\title{
A NEW HETEROGENEOUS MULTISCALE METHOD FOR TIME-HARMONIC MAXWELL'S EQUATIONS*
}

\author{
PATRICK HENNING ${ }^{\dagger}$, MARIO OHLBERGER ${ }^{\ddagger}$, AND BARBARA VERFÜRTH ${ }^{\ddagger}$
}

\begin{abstract}
In this paper, we suggest a new heterogeneous multiscale method (HMM) for the time-harmonic Maxwell equations in locally periodic media. The method is constructed by using a divergence-regularization in one of the cell problems. This allows us to introduce fine-scale correctors that are not subject to a cumbersome divergence-free constraint and which can hence easily be implemented. To analyze the method, we first revisit classical homogenization theory for time-harmonic Maxwell equations and derive a new homogenization result that makes use of the divergence-regularization in the two-scale homogenized equation. We then show that the HMM is equivalent to a discretization of this equation. In particular, writing both problems in a fully coupled two-scale formulation is the crucial starting point for a corresponding numerical analysis of the method. With this approach we are able to prove rigorous a priori error estimates in the $\mathbf{H}$ (curl)and the $H^{-1}$-norm, and we derive reliable and efficient localized residual-based a posteriori error estimates. Numerical experiments are presented to verify the a priori convergence results.
\end{abstract}

Key words. multiscale method, finite elements, Maxwell's equations, homogenization, two-scale convergence

AMS subject classifications. 35B27, 65N15, 65N30, 78M40

DOI. $10.1137 / 15 \mathrm{M} 1039225$

1. Introduction. The behavior and propagation of electromagnetic fields is studied in many physical applications, for instance, in the large area of wave optics. Periodic and locally periodic materials are considered with growing interest, for example, in the application of photonic crystals (see [33] for an introduction), as they can show unusual behavior, such as photonic band gaps and even negative refraction (see, e.g., $[19,35,39,42])$. However, a thorough mathematical understanding of these phenomena is still lacking. Therefore, one major goal is to develop efficient numerical schemes to simulate wave propagation in periodic materials and to rigorously analyze the new algorithms and the errors they introduce.

Electromagnetic problems are governed by Maxwell's equations. We consider a linear conductive medium, subject to Ohm's law. We study the time-harmonic case, i.e., all quantities are of the form $\hat{\psi}(x, t)=\operatorname{Re}\left(\psi(x) e^{i \omega t}\right)$ with a complex-valued function $\psi$ and a temporal frequency $\omega \neq 0$. Maxwell's equations can then be reduced to the following linear curl-curl-problem: Let $\Omega \subset \mathbb{R}^{3}$ be a bounded domain with outer normal $\mathbf{n}$ on $\partial \Omega$, and we seek the electric field $\mathbf{E}_{\delta}: \Omega \rightarrow \mathbb{C}^{3}$ with

$$
\begin{aligned}
\operatorname{curl}\left(\mu_{\delta}^{-1} \operatorname{curl} \mathbf{E}_{\delta}\right)-\kappa_{\delta} \mathbf{E}_{\delta} & =\mathbf{f} & & \text { in } \Omega, \\
\mathbf{E}_{\delta} \times \mathbf{n} & =0 & & \text { on } \partial \Omega .
\end{aligned}
$$

${ }^{*}$ Received by the editors September 11, 2015; accepted for publication (in revised form) September 9, 2016; published electronically December 1, 2016.

http://www.siam.org/journals/sinum/54-6/M103922.html

Funding: This work was supported by the Deutsche Forschungsgemeinschaft (DFG) in the project "OH 98/6-1: Wellenausbreitung in periodischen Strukturen und Mechanismen negativer Brechung."

$\dagger$ Division of Numerical Analysis, Mathematics, Kungliga Tekniska Högeskolan, 10044 Stockholm, Sweden (pathe@kth.se).

‡Institut für Numerische und Angewandte Mathematik, Westfälische Wilhelms-Universität Münster, D-48149 Münster, Germany (mario.ohlberger@uni-muenster.de, barbara.verfuerth@unimuenster.de). 
Here, $\mu^{-1}$ is the inverse permeability, $\kappa=\omega^{2} \varepsilon-i \omega \sigma$ is related to the permittivity $\varepsilon$ and the conductivity $\sigma$, and the right-hand side $\mathbf{f}=-i \omega \mathbf{j}$ models the (source) current density. The magnetic field $\mathbf{H}$ can be computed as $\mathbf{H}=i \omega^{-1} \mu^{-1} \operatorname{curl} \mathbf{E}$. We will assume that the real-valued parameter $\mu_{\delta}^{-1}$ and the complex-valued parameter $\kappa_{\delta}$ are locally periodic with periodicity length $\delta$, where $\delta$ is very small compared to $\Omega$ and the wavelength $\lambda \sim 1 / \omega$. The boundary condition (1.2) models the case where $\Omega$ is surrounded by a so-called perfect electric conductor. We refer to $[37,41]$ or $[48]$ for a detailed motivation and further applications.

Since a numerical treatment of (1.1)-(1.2) requires discretizations with mesh sizes $h<\delta \ll 1$, corresponding computations can easily exceed today's available computer resources if tackled with a standard approach. In order to make the problem numerically solvable, so-called multiscale methods can be applied. One class of multiscale methods that has been proved to be very efficient for scale-separated problems with local periodicity (or mild heterogeneities) is the family of heterogenous multiscale methods (HMM) introduced by Engquist and E [20, 21]. HMM approaches exploit structural invariants in the coefficients to solve local sample problems that allow to extract representative features. Therefore, for scale-separated and locally periodic problems, the $\delta$-dependent multiscale solution can then be approximated with a computational complexity that is, however, independent of $\delta$. With this strategy the problem becomes solvable even for arbitrarily small values of $\delta$. First analytical results concerning the approximation properties of the HMM for diffusion problems have been derived in $[1,18,26,38]$. In this contribution we formulate and analyze a new HMM for solving the curl-curl-problem (1.1)-(1.2).

Concerning wave propagation in general, the HMM and other multiscale methods for wave equations have been studied by Abdulle and co-authors [2, 3], Engquist, Runborg, and co-authors [6, 22, 23, 24], and Jiang, Efendiev, and co-authors [32, 31]. An HMM for the Helmholtz equation has been suggested in [13]. Furthermore, some methods based on asymptotic expansions have been suggested for Maxwell's equations; see, e.g., [11, 49]. Finally, a similar HMM for Maxwell's equations in frequency domain has been outlined in [14], but with a different approach to the a priori error analysis and without a posteriori estimates.

The new contribution of this article is the first formulation of an HMM for the time-harmonic Maxwell equations and its comprehensive numerical analysis in terms of a priori and a posteriori error estimates. The error analysis can serve as a starting point for a locally mesh adaptive version of the described HMM.

The idea of the HMM is to adapt the (analytical) homogenization procedure to the numerical scheme. Therefore, we will first have a look at the homogenization of the time-harmonic Maxwell equations. Combining results by Wellander and co-authors [45, 46, 47] and Visintin [44], we derive a new two-scale equation for timeharmonic Maxwell's equations. One essential step in the homogenization procedure is a divergence-regularization in order to incorporate a divergence-free constraint imposed on the corrector of the curl into the equation. This regularization also is an essential ingredient in the formulation of the new HMM. We will then adopt the view of the HMM as a direct discretization of the derived two-scale equation. This reformulation builds the crucial ingredient for an a posteriori analysis. It has been first developed in [38] and has then been adopted to other problems, as perforated domains [28] or advection-diffusion problems [29], for instance. There have been several contributions on the numerical analysis for time-harmonic Maxwell's equations. (See the excellent book [37] for an overview, [48] for higher order finite elements, and $[40,10]$ for a posteriori analysis.) In the analysis of our HMM, we combine these error 
estimation techniques for multiscale methods and for Maxwell's equations. Thereby, we are able to prove $\delta$-independent error estimates that a rate-optimal (with respect to mesh refinement) for sufficiently smooth solutions.

The article is organized as follows: In section 2 we formulate the multiscale curlcurl-problem and give some properties of the solution. The problem is homogenized with the tool of two-scale convergence in section 3. The homogenized formulation is the motivation and starting point for the formulation of the HMM in section 4. Error estimates for this method are given in section 5. All essential proofs are detailed in section 6 . We verify the theoretical results by a numerical example in section 7 .

2. Problem setting. For the remainder of this article, let $\Omega \subset \mathbb{R}^{3}$ be a bounded, simply connected domain with connected piecewise polygonal Lipschitz boundary $\partial \Omega$ and outer unit normal $\mathbf{n}$. Throughout this paper, we use standard notation: By $W^{l, p}(\Omega)$ we denote the space of functions on $\Omega$ with weak derivatives up to order $l$ belonging to $L^{p}(\Omega)$, and we write $H^{l}(\Omega):=W^{l, 2}(\Omega)$ for the scalar and $\mathbf{H}^{l}(\Omega):=$ $\left[H^{l}(\Omega)\right]^{3}$ for the vector-valued case. Vector-valued functions are indicated by boldface letters, and unless otherwise stated, all functions are complex-valued. The dot will denote a normal (real) scalar product; for a complex scalar product we will explicitly conjugate the second component by using $u^{*}$ as the complex conjugate of $u$. For any domain $\omega \subset \mathbb{R}^{3}$, we introduce the spaces

$$
\begin{aligned}
\mathbf{H}(\operatorname{curl}, \omega) & :=\left\{\mathbf{u} \in L^{2}\left(\omega ; \mathbb{C}^{3}\right) \mid \operatorname{curl} \mathbf{u} \in L^{2}\left(\omega ; \mathbb{C}^{3}\right)\right\} \quad \text { and } \\
\mathbf{H}(\operatorname{div}, \omega) & :=\left\{\mathbf{u} \in L^{2}\left(\omega ; \mathbb{C}^{3}\right) \mid \operatorname{div} \mathbf{u} \in L^{2}(\omega ; \mathbb{C})\right\} .
\end{aligned}
$$

For $\omega=\Omega$ we write $\mathbf{H}(\operatorname{curl}):=\mathbf{H}(\operatorname{curl}, \Omega)$ and $\mathbf{H}(\operatorname{div}):=\mathbf{H}(\operatorname{div}, \Omega)$. These spaces are complex Hilbert spaces if endowed with the scalar products

$$
\begin{aligned}
(\mathbf{u}, \mathbf{v})_{\mathbf{H}(\operatorname{curl})} & :=\int_{\Omega} \operatorname{curl} \mathbf{u} \cdot \operatorname{curl} \mathbf{v}^{*}+\mathbf{u} \cdot \mathbf{v}^{*} d x, \\
(\mathbf{u}, \mathbf{v})_{\mathbf{H}(\operatorname{div})} & :=\int_{\Omega} \operatorname{div} \mathbf{u} \operatorname{div} \mathbf{v}^{*}+\mathbf{u} \cdot \mathbf{v}^{*} d x
\end{aligned}
$$

Zero boundary values (in the sense of traces) for functions in $\mathbf{H}$ (curl) can be defined as (see [37])

$$
\mathbf{H}_{0}(\operatorname{curl}):=\{\mathbf{v} \in \mathbf{H}(\operatorname{curl}) \mid \mathbf{v} \times \mathbf{n}=0\} .
$$

To quantify higher regularity, we define for $s \in \mathbb{N}_{0}$ the space

$$
\mathbf{H}^{s}(\operatorname{curl}):=\left\{\mathbf{u} \in \mathbf{H}(\operatorname{curl}) \mid \mathbf{u} \in \mathbf{H}^{s}(\Omega), \operatorname{curl} \mathbf{u} \in \mathbf{H}^{s}(\Omega)\right\} .
$$

Observe that $\mathbf{H}^{0}$ (curl) $=\mathbf{H}\left(\right.$ curl). Let $\mathbf{e}_{k}$ denote the $k$ th unit vector in $\mathbb{R}^{3}$. For the rest of the paper we write $Y:=\left[-\frac{1}{2}, \frac{1}{2}\right)^{3}$ to denote the three-dimensional unit cube, and we say that a function $v \in L_{\text {loc }}^{2}\left(\mathbb{R}^{3}\right)$ is $Y$-periodic if it fulfills $v(y)=v\left(y+\mathbf{e}_{k}\right)$ for all $k=1,2,3$ and almost every $y \in \mathbb{R}^{3}$. With that we denote $L_{\sharp}^{2}(Y):=\{v \in$ $L_{\text {loc }}^{2}\left(\mathbb{R}^{3}\right) \mid v$ is $Y$-periodic $\}$. Analogously we indicate periodic function spaces by the subscript $\sharp$. For example, $H_{\sharp}^{1}(Y)$ is the space of periodic $H_{\text {loc }}^{1}\left(\mathbb{R}^{3}\right)$-functions, and we furthermore define for $s \in \mathbb{N}$

$$
H_{\sharp, 0}^{s}(Y):=\left\{\phi \in H_{\sharp}^{s}(Y) \mid \int_{Y} \phi(y) d y=0\right\} .
$$

By $L^{p}(\Omega ; X)$ we denote Bochner-Lebesgue spaces over the Banach space $X$, and we use the short notation $f(x, y):=f(x)(y)$ for $f \in L^{p}(\Omega ; X)$.

Using the above notation we make the following assumptions on the data. 
Assumption 2.1. Let $\mathbf{f} \in \mathbf{H}($ div $)$ with $\operatorname{div} \mathbf{f}=0$. Define $\kappa_{\delta}(x):=\kappa\left(x, \frac{x}{\delta}\right)$, $\mu_{\delta}^{-1}(x):=\mu^{-1}\left(x, \frac{x}{\delta}\right)$, where the scalar coefficients $\mu^{-1}$ and $\kappa$ fulfill

- $\mu^{-1} \in C^{0}\left(\Omega ; L_{\sharp}^{\infty}(Y)\right)$ is real-valued,

- $\kappa \in C^{0}\left(\Omega ; L_{\sharp}^{\infty}(Y ; \mathbb{C})\right)$ is complex-valued,

- $\mu^{-1}$ is bounded and strictly positive, uniform in $x$ and $y$,

- $\operatorname{Re}(\kappa)$ is bounded and strictly positive, uniform in $x$ and $y$,

- $\operatorname{Im}(\kappa)$ is bounded and strictly negative, uniform in $x$ and $y$.

$\operatorname{div} \mathbf{f}=0$ can be justified by physics but is only assumed for simplicity. Up to some minor modifications, all presented results also hold without that assumption. The assumptions of boundedness and positivity/negativity on $\kappa$ mean that the permittivity $\varepsilon$ and the conductivity $\sigma$ are bounded and strictly positive functions and that the frequency $\omega$ is bounded. Furthermore, our homogenization approach is only justified in the regime where $\delta$ is small in comparison to the wavelength $\lambda \sim \frac{1}{\omega \sqrt{\mu \varepsilon}}$ and the skin layer length $l_{s} \sim \frac{1}{\sqrt{\omega \sigma \mu}}$; see [5] for details. Roughly speaking, this means that we assume the so-called low-frequency case with $\delta \ll 1 / \omega$ for the whole paper. The regularity assumptions on $\mu^{-1}$ and $\kappa$ imply that these functions are admissible test functions for two-scale convergence in the sense of Allaire; see [4, Definition 1.4 and Corollary 5.4]. This is needed for the homogenization results in section 3.

We look for the weak solution of (1.1)-(1.2): Let Assumption 2.1 be fulfilled and find $\mathbf{E}_{\delta} \in \mathbf{H}_{0}$ (curl) such that, for all $\boldsymbol{\psi} \in \mathbf{H}_{0}$ (curl),

$$
\int_{\Omega} \mu_{\delta}^{-1}(x) \operatorname{curl} \mathbf{E}_{\delta}(x) \cdot \operatorname{curl} \boldsymbol{\psi}^{*}(x)-\kappa_{\delta} \mathbf{E}_{\delta}(x) \cdot \boldsymbol{\psi}^{*}(x) d x=\int_{\Omega} \mathbf{f}(x) \cdot \boldsymbol{\psi}^{*}(x) d x .
$$

For fixed $\delta$, there is a unique solution to $(2.2)$, which can be seen using the Lax-Milgram-Babuška theorem, [7]: Clearly, the right-hand side is a member of the dual space and the left-hand side gives a continuous sesquilinear form. Since $\operatorname{Im} \kappa$ is bounded away from zero, we also get the coercivity estimate $\left|B_{\delta}(\mathbf{u}, \mathbf{u})\right| \geq C\|\mathbf{u}\|_{\mathbf{H} \text { (curl) }}^{2}$ with a $\delta$-independent constant. See [48] for the case of constant coefficients and [25] for the general computation. Hence, we also have the uniform estimate $\left\|\mathbf{E}_{\delta}\right\|_{\mathbf{H} \text { (curl) }} \leq$ $C\|\mathbf{f}\|_{L^{2}}$ with $C=C\left(\mu^{-1}, \kappa, \Omega\right)$.

In general, solutions to curl-curl-problems do not admit more than $H^{1 / 2}(\Omega)$ regularity and may have singularities near reentrant corners of the domain; see [16]. However, if $\Omega$ is convex and if $\mu^{-1}, \kappa \in W^{1, \infty}(\Omega \times Y)$, i.e., the coefficients are globally Lipschitz, the weak solution to (2.2) fulfills $\mathbf{E}_{\delta} \in \mathbf{H}^{1}$ (curl); see [41].

3. Homogenization. As the periodicity length $\delta$ is assumed to be very small in comparison to $\Omega$, one can reduce the complexity of the problem by considering the limit $\delta \rightarrow 0$. This process is called homogenization and can be performed with the tool of two-scale convergence [4]. Since the two-scale equation and the homogenized equation are the starting point for the construction and analysis of the numerical multiscale method later on, we present the essential steps in this section.

3.1. Two-scale convergence. Two-scale convergence is a special form of convergence for locally periodic functions, which tries to capture oscillations. For its definition and further details, such as the definition of strong two-scale convergence and compactness results in $L^{2}(\Omega)$ or $H^{1}(\Omega)$, we refer the reader to [4,34] and the lecture script [27].

For time-harmonic Maxwell's equations we need a two-scale convergence result for bounded sequences in $\mathbf{H}$ (curl). As $\mathbf{H}$ (curl) is not compactly embedded in $L^{2}(\Omega)$ (in 
contrast to $H^{1}(\Omega)$ ), the two-scale limit in $L^{2}$ will not coincide with the weak limit, thus making additional considerations necessary (cf. [44, p. 135]). We have the following result for two-scale convergence in $\mathbf{H}$ (curl) from the literature [12, 44, 45, 46, 47].

Proposition 3.1. Let $\left(\mathbf{u}_{\delta}\right)_{\delta>0} \subset \mathbf{H}($ curl $)$ be a bounded sequence. Then there exists a subsequence and functions $\mathbf{u}_{0} \in L^{2}(\Omega \times Y), \mathbf{u}_{1} \in L^{2}\left(\Omega ; H_{\sharp, 0}^{1}(Y)\right)$ with $\operatorname{div}_{y} \mathbf{u}_{1}=0$ a.e. and $\phi \in L^{2}\left(\Omega ; H_{\sharp, 0}^{1}(Y)\right)$ such that

1. $\mathbf{u}_{\delta} \stackrel{2}{\rightarrow} \mathbf{u}_{0}$ with $\mathbf{u}_{0}(x)=\mathbf{u}(x)+\nabla_{y} \phi(x, y)$ and $\mathbf{u}=\int_{Y} \mathbf{u}_{0}(\cdot, y) d y \in \mathbf{H}(\operatorname{curl})$,

2. $\operatorname{curl} \mathbf{u}_{\delta} \stackrel{2}{\rightarrow} \operatorname{curl} \mathbf{u}+\operatorname{curl}_{y} \mathbf{u}_{1}$.

Note that the condition $\operatorname{div}_{y} \mathbf{u}_{1}=0$ can be seen as a kind of gauging condition. It will be important for the homogenization of our curl-curl-problem, namely, this condition will lead to the uniqueness of the two-scale solution.

3.2. Homogenization for time-harmonic Maxwell's equations. In this section we present new homogenization results for the time-harmonic Maxwell equations in a two-scale formulation, a formulation with cell problems and macroscopic equations, and a corrector result. We emphasize that although Maxwell's equations and curl-curl-problems have been homogenized in the literature $([5,45,47]$ to name only a few), the focus has mostly been on macroscopic (homogenized) problems as (3.5), but not on two-scale limit equations. A two-scale result similar to the following theorem has been presented in [47], but for the coupled system of first order equations the electric and magnetic field, and recently in [12]. The first one includes some incorrect terms as remarked in [46].

Theorem 3.2 (two-scale equation). Under Assumption 2.1, let $\mathbf{E}_{\delta} \in \mathbf{H}_{0}$ (curl) be a solution of (2.2). Then there exists a solution triple $\left(\mathbf{E}, \mathbf{K}_{1}, K_{2}\right)$ of functions $\mathbf{E} \in \mathbf{H}_{0}(\operatorname{curl}), \mathbf{K}_{1} \in L^{2}\left(\Omega ; \mathbf{H}_{\sharp, 0}^{1}(Y)\right)$ with $\operatorname{div}_{y} \mathbf{K}_{1}=0$ a.e., and $K_{2} \in L^{2}\left(\Omega ; H_{\sharp, 0}^{1}(Y)\right)$ such that

$$
\mathbf{E}_{\delta} \rightarrow \mathbf{E} \text { in } \mathbf{H}_{0}(\operatorname{curl}), \quad \mathbf{E}_{\delta} \stackrel{2}{\rightarrow} \mathbf{E}+\nabla_{y} K_{2}, \quad \operatorname{curl} \mathbf{E}_{\delta} \stackrel{2}{\rightarrow} \operatorname{curl} \mathbf{E}+\operatorname{curl}_{y} \mathbf{K}_{1} .
$$

Considered in $\mathbf{H}_{0}(\operatorname{curl}) \times L^{2}\left(\Omega ; \mathbf{H}_{\sharp, 0}^{1}(Y)\right) \times L^{2}\left(\Omega ; H_{\sharp, 0}^{1}(Y)\right)$, the triple $\left(\mathbf{E}, \mathbf{K}_{1}, K_{2}\right)$ is the unique solution of

$$
\begin{aligned}
& \mathcal{B}\left(\left(\mathbf{E}, \mathbf{K}_{1}, K_{2}\right),\left(\boldsymbol{\psi}, \boldsymbol{\psi}_{1}, \psi_{2}\right)\right)=\int_{\Omega} \mathbf{f}(x) \cdot \boldsymbol{\psi}^{*}(x) d x \\
& \quad \forall \boldsymbol{\psi} \in \mathbf{H}_{0}(\operatorname{curl}), \boldsymbol{\psi}_{1} \in L^{2}\left(\Omega ; \mathbf{H}_{\sharp, 0}^{1}(Y)\right), \psi_{2} \in L^{2}\left(\Omega ; H_{\sharp, 0}^{1}(Y)\right)
\end{aligned}
$$

with the two-scale sesquilinear form $\mathcal{B}$ defined by

$$
\begin{aligned}
\mathcal{B}\left(\left(\mathbf{u}, \mathbf{u}_{1}, u_{2}\right),\left(\boldsymbol{\psi}, \boldsymbol{\psi}_{1}, \psi_{2}\right)\right) & \\
:=\int_{\Omega} \int_{Y} & \mu^{-1}(x, y)\left(\operatorname{curl} \mathbf{u}(x)+\operatorname{curl}_{y} \mathbf{u}_{1}(x, y)\right) \cdot\left(\operatorname{curl} \boldsymbol{\psi}^{*}(x)+\operatorname{curl}_{y} \boldsymbol{\psi}_{1}^{*}(x, y)\right) \\
& +\operatorname{div}_{y} \mathbf{u}_{1}(x, y) \operatorname{div}_{y} \boldsymbol{\psi}_{1}^{*}(x, y) \\
& \quad \kappa(x, y)\left(\mathbf{u}(x)+\nabla_{y} u_{2}(x, y)\right) \cdot\left(\boldsymbol{\psi}^{*}(x)+\nabla_{y} \psi_{2}^{*}(x, y)\right) d y d x
\end{aligned}
$$

The proof is postponed to section 6 .

In order to determine $\mathbf{K}_{1}$ in the two-scale equation, one has to solve the following problem: Find $\mathbf{u} \in \mathbf{H}(\operatorname{curl}, Y) \cap \mathbf{H}(\operatorname{div}, Y)$ with $\operatorname{div} \mathbf{u}=0$ a.e. in $Y$ such that $\int_{Y} \mu^{-1} \operatorname{curl} \mathbf{u} \cdot \operatorname{curl} \boldsymbol{\psi}^{*} d y=0$ for all $\boldsymbol{\psi} \in \mathbf{H}(\operatorname{curl}, Y)$ with appropriate boundary conditions. The divergence-free constraint $\operatorname{div} \mathbf{u}=0$ is necessary to guarantee the unique- 
ness of a solution, as otherwise the solution is only determined up to a gradient term. However, divergence-free finite elements are quite rare and thus, the constraint has to be fulfilled implicitly in the equation.

With divergence-regularization, we now look for $\mathbf{u} \in \mathbf{H}^{1}(Y)$ such that

$$
\int_{Y} \mu^{-1} \operatorname{curl} \mathbf{u} \cdot \operatorname{curl} \boldsymbol{\psi}^{*}+\operatorname{div} \mathbf{u} \operatorname{div} \boldsymbol{\psi}^{*} d y=0 \quad \forall \boldsymbol{\psi} \in \mathbf{H}^{1}(Y) .
$$

Both problems are equivalent for convex domains; see [16]. This geometrical condition is no constraint here as the corrector problem is always posed on the obviously convex unit cube $Y$. There are other possibilities to deal with a divergence-free constraint. The introduction of Lagrange multipliers (see [16]) leads to a mixed problem, which increases the computational costs and complicates the error analysis. The $s$-regularization suggested in [17] makes the reformulation of the HMM later on (Proposition 4.3) impossible, since different orders of derivatives appear.

DEFINITION 3.3 (cell problems and homogenized matrices). The cell problems are to find functions $\mathbf{v}_{k} \in L^{2}\left(\Omega ; \mathbf{H}_{\sharp, 0}^{1}(Y)\right), v_{k} \in L^{2}\left(\Omega ; H_{\sharp, 0}^{1}(Y)\right)$ so that a.e. in $\Omega$ there holds

$$
\begin{array}{cl}
\int_{Y} \mu^{-1}(x, y)\left(\mathbf{e}_{k}+\operatorname{curl}_{y} \mathbf{v}_{k}(x, y)\right) \cdot \operatorname{curl} \boldsymbol{\psi}^{*}(y) & \\
+\operatorname{div}_{y} \mathbf{v}_{k}(x, y) \operatorname{div} \boldsymbol{\psi}^{*}(y) d y=0 & \forall \boldsymbol{\psi} \in \mathbf{H}_{\sharp, 0}^{1}(Y), \\
\int_{Y} \kappa(x, y)\left(\mathbf{e}_{k}+\nabla_{y} v_{k}(x, y)\right) \cdot \nabla \psi^{*}(y) d y=0 & \forall \psi \in H_{\sharp, 0}^{1}(Y) .
\end{array}
$$

With the (unique) solutions of the cell problems (3.2)-(3.3) we define the homogenized matrices

$$
\begin{aligned}
\left(\mu^{-1}\right)_{j, k}^{\mathrm{hom}}(x) & =\int_{Y} \mu^{-1}(x, y)\left(\operatorname{Id}_{j k}+\left(\operatorname{curl}_{y} \mathbf{v}_{k}(x, y)\right)_{j}\right) d y \\
\kappa_{j, k}^{\text {hom }}(x) & =\int_{Y} \kappa(x, y)\left(\operatorname{Id}_{j k}+\left(\nabla_{y} v_{k}(x, y)\right)_{j}\right) d y, \quad j, k=1,2,3 .
\end{aligned}
$$

The homogenized matrices are used to formulate the macroscale problem for $\mathbf{E}$. It has the same structure as our original problem except that the material parameters are now matrices and not scalar functions.

Theorem 3.4 (equivalence of two-scale and homogenized equation). The triple $\left(\mathbf{E}, \mathbf{K}_{1}, K_{2}\right)$ is the unique solution of (3.1) iff $\mathbf{E} \in \mathbf{H}_{0}$ (curl) solves

$$
\int_{\Omega}\left(\mu^{-1}\right)^{\mathrm{hom}} \operatorname{curl} \mathbf{E} \cdot \operatorname{curl} \boldsymbol{\psi}^{*}-\kappa^{\mathrm{hom}} \mathbf{E} \cdot \boldsymbol{\psi}^{*} d x=\int_{\Omega} \mathbf{f} \cdot \boldsymbol{\psi}^{*} d x
$$

for all $\boldsymbol{\psi} \in \mathbf{H}_{0}$ (curl) with the matrices $\left(\mu^{-1}\right)^{\text {hom }}, \kappa^{\text {hom }}$ defined through (3.4) and with correctors $\mathbf{K}_{1}, K_{2}$ defined as $\mathbf{K}_{1}(x, y)=\sum_{k=1}^{3}(\operatorname{curl} \mathbf{E}(x))_{k} \mathbf{v}_{k}(x, y), K_{2}(x, y)=$ $\sum_{k=1}^{3} \mathbf{E}_{k}(x) v_{k}(x, y)$, where $\mathbf{v}_{k}, v_{k}$ are solutions of the cell problems (3.2), (3.3).

Proof. Inserting the cell problems and the definition of the homogenized matrices into (3.5) leads to the two-scale equation.

We end this section by a corrector-type result, which relates the two-scale solution to the asymptotic expansion. The assumption in the theorem below can, for instance, be fulfilled if the mentioned functions belong to $C^{0}\left(\bar{\Omega} ; L_{\sharp}^{2}(Y)\right)$, which is a 
regularity assumption on the geometry and the material parameters. Other functions spaces giving admissible test functions for two-scale convergence are mentioned in [34, Theorem 3]. See also the related corrector result in [47] for the coupled first order system of equations for the electric and magnetic field.

THEOREM 3.5 (strong convergence in $\mathbf{H}\left(\right.$ curl)). Let $\mu^{-1}, \kappa, \mathbf{K}_{1}, \operatorname{curl}_{x} \mathbf{K}_{1}$, $\operatorname{curl}_{y} \mathbf{K}_{1}, \nabla_{x} K_{2}$, and $\nabla_{y} K_{2}$ be admissible test functions for two-scale convergence in the sense of Allaire [4, Def. 1.4]. Then it holds that

$$
\left\|\mathbf{E}_{\delta}(x)-\left(\mathbf{E}(x)+\delta\left(\mathbf{K}_{1}\left(x, \frac{x}{\delta}\right)+\nabla K_{2}\left(x, \frac{x}{\delta}\right)\right)\right)\right\|_{\mathbf{H}(\text { curl })} \stackrel{\delta \rightarrow 0}{\longrightarrow} 0 .
$$

Proof. Inserting the term in the norm into the heterogeneous sesquilinear form $B_{\delta}$ and using the chain rule and two-scale convergence gives the claim.

The theorem shows that the correctors $\mathbf{K}_{1}$ and $K_{2}$ represent a Helmholtz decomposition of the first order term in the asymptotic expansion. Since on the gradient subspace, the $\mathbf{H}$ (curl)-norm and the $L^{2}$-norm are equivalent, we see that in particular $K_{2}$ carries important information about the solution $\mathbf{E}_{\delta}$ itself. Thus, in contrast to the case of diffusion problems, the correctors $\mathbf{K}_{1}, K_{2}$ have to be considered as well (and not only the weak limit $\mathbf{E}$ ) in order to get a good $L^{2}$-approximation of the heterogeneous solution $\mathbf{E}_{\delta}$. This is a crucial observation. Consequently, the HMM is not only constructed to approximate $\mathbf{E}$ but requires to approximate $\mathbf{K}_{1}$ and $K_{2}$ as well. Only recently, a $\delta$-explicit estimate for the homogenization error has been proved in [12]. Assuming sufficient regularity of the data and the analytical two-scale solution (for details see [12]), we have (see [12, Theorem 3.1] and the proof thereof)

$$
\left\|\mathbf{E}_{\delta}-\left(\mathbf{E}+\delta\left(\mathbf{K}_{1}\left(\cdot, \frac{\dot{\delta}}{\delta}\right)+\nabla K_{2}\left(\cdot, \frac{\dot{\delta}}{\delta}\right)\right)\right)\right\|_{\mathbf{H}(\text { curl })} \leq C \delta^{1 / 2} .
$$

4. The heterogeneous multiscale method. The basic idea of the HMM is to use a macroscopic sesquilinear form similar to (3.5) for the finite element method. Instead of solving the cell problems on the unit cube, local variants are set up and solved around the centers of the tetrahedra of some macroscopic computational grid. In order to define the method in more detail, let us introduce some definitions.

Denote by $\mathcal{T}_{H}=\left\{T_{j} \mid j \in J\right\}$ and $\mathcal{T}_{h}=\left\{S_{k} \mid k \in I\right\}$ regular (i.e., without hanging nodes or edges) and shape regular, simplicial partitions of $\Omega$ and $Y$, respectively. Additionally, we assume that $\mathcal{T}_{h}$ is periodic in the sense that it can be wrapped to a regular simplicial partition of the torus, i.e., no hanging nodes or edges occur over the periodic boundary. The $\delta$-scaled and $x_{j}$-shifted unit cubes are denoted by $Y_{j}^{\delta}=\delta Y+x_{j}$, together with the mappings $y_{j}^{\delta}: Y_{j}^{\delta} \rightarrow Y$ and $x_{j}^{\delta}=\left(y_{j}^{\delta}\right)^{-1}: Y \rightarrow Y_{j}^{\delta}$. A triangulation of the shifted unit cubes is then given by $\mathcal{T}_{h}\left(Y_{j}^{\delta}\right)=\left\{\tilde{S} \mid \tilde{S}=x_{j}^{\delta}(S), S \in\right.$ $\left.\mathcal{T}_{h}\right\}$. The set of interior faces is defined as

$$
\mathcal{E}\left(\mathcal{T}_{H}\right)=\left\{(j, l) \in J \times J \mid F_{j l}:=\bar{T}_{j} \cap \bar{T}_{l} \neq \emptyset, \operatorname{dim}\left(F_{j l}\right)=2, j<l\right\}
$$

and $\mathcal{E}\left(\mathcal{T}_{h}\right)$ with the faces $\tilde{F}_{i k}$ is defined analogously. The direct neighbors of a face $F_{j l}$ are $\hat{\omega}_{F_{j l}}:=T_{j} \cup T_{l}$. The neighborhoods of vertices $V$, faces $F$, and elements $T$ are defined as

$$
\omega_{V}:=\bigcup_{j \in J, V \in \bar{T}_{j}} \bar{T}_{j}, \quad \omega_{F_{j l}}:=\bigcup_{V \in F_{j l}} \omega_{V}, \quad \omega_{T_{j}}:=\bigcup_{V \in \bar{T}_{j}} \omega_{V}
$$


and the neighborhood of neighbors of an element is defined as

$$
\tilde{\omega}_{T_{j}}:=\bigcup_{V \in \bar{T}_{j}} \bigcup_{V^{\prime} \in \omega_{V}} \omega_{V^{\prime}}
$$

We define the local mesh sizes $H_{j}:=\operatorname{diam}\left(T_{j}\right), h_{k}:=\operatorname{diam}\left(S_{k}\right), H_{j l}:=$ $\operatorname{diam}\left(F_{j l}\right), h_{k m}:=\operatorname{diam}\left(\tilde{F}_{k m}\right)$, and the global mesh sizes $H:=\max _{j \in J} H_{j}$ and $h:=\max _{k \in I} h_{k}$. Note that $h$ denotes the mesh width of the partition of the unit cube $Y$. We stress that it is in no way related to $\delta$ and can be of the same order as $H$. The $\delta$-shifted cubes $Y_{j}^{\delta}$, where the actual (local) finescale computations are carried out, consequently have a mesh size of $\delta h$.

Finally, the discrete function spaces $\mathbf{V}_{H, 0}^{I} \subset \mathbf{H}_{0}(\operatorname{curl})$ and $\widetilde{W}_{h}^{1}\left(Y_{j}^{\delta}\right) \subset H_{\sharp, 0}^{1}\left(Y_{j}^{\delta}\right)$ are defined as

$$
\begin{aligned}
\mathbf{V}_{H, 0}^{I} & :=\left\{\mathbf{u}_{H} \in \mathbf{H}_{0}(\operatorname{curl})\left|\mathbf{u}_{h}\right|_{T} \in N_{0} \quad \forall T \in \mathcal{T}_{H}\right\}, \\
\widetilde{W}_{h}^{1}\left(Y_{j}^{\delta}\right) & :=\left\{u_{h} \in H_{\sharp, 0}^{1}\left(Y_{j}^{\delta}\right)\left|u_{h}\right|_{S} \in \mathbb{P}^{1} \quad \forall S \in \mathcal{T}_{h}\left(Y_{j}^{\delta}\right)\right\},
\end{aligned}
$$

where $\mathbb{P}^{1}$ are the polynomials of maximal degree 1 and $N_{0}$ is the lowest order Nédélec element of the first family, given by $N_{0}:=\left\{\mathbf{a} \times x+\mathbf{b} \mid \mathbf{a}, \mathbf{b} \in \mathbb{C}^{3}\right\}$. As in the analytical case, bold face letters indicate vector-valued functions and function spaces, for instance, $\widetilde{\mathbf{W}}_{h}^{1}:=\left(\widetilde{W}_{h}^{1}\right)^{3}$. We pick numerical quadrature rules that are exact for the given test and ansatz spaces: In our case of piecewise linear functions, it suffices to choose the one-point rule $\left\{x_{j},\left|T_{j}\right|\right\}$ with the barycenter $x_{j}$ for the curl-part and a second order quadrature rule $Q_{j}^{(2)}:=\left\{q_{l}, x_{l}\right\}_{l}$ with $l=1, \ldots, 4$ for the identity part.

With these preliminaries we can now define the HMM (see also [20, 21, 38]).

DeFINITION 4.1 (HMM). The HMM-approximation of (2.2) is a discrete solution triple $\left(\mathbf{E}_{H}, \mathbf{R}_{1}\left(\mathbf{E}_{H}\right), \mathbf{R}_{2}\left(\mathbf{E}_{H}\right)\right)$, where $\mathbf{E}_{H} \in \mathbf{V}_{H, 0}^{I}$ is defined as the solution of

$$
B_{H}\left(\mathbf{E}_{H}, \boldsymbol{\psi}_{H}\right)=\left(f, \boldsymbol{\psi}_{H}\right) \quad \forall \boldsymbol{\psi}_{H} \in \mathbf{V}_{H, 0}^{I},
$$

where the discrete sesquilinear form is given by

$$
\begin{aligned}
B_{H}\left(\mathbf{u}_{H}, \boldsymbol{\psi}_{H}\right):= & \sum_{j \in J} \frac{\left|T_{j}\right|}{\delta^{3}} \int_{Y_{j}^{\delta}}\left(\mu^{-1}\right)_{h}^{\delta}(x) \operatorname{curl} \mathbf{R}_{j, 1}\left(\mathbf{u}_{H}\right)(x) \cdot \operatorname{curl} \boldsymbol{\psi}_{H}^{*}(x) \\
& -\sum_{j \in J} \sum_{l \in Q_{j}^{(2)}} \frac{q_{l}}{\delta^{3}} \int_{Y_{l}^{\delta}} \kappa_{h}^{\delta}(x) \mathbf{R}_{l, 2}\left(\mathbf{u}_{H}\right)(x) \cdot \mathbf{R}_{l, 2}\left(\boldsymbol{\psi}_{H}\right)^{*}(x) d x
\end{aligned}
$$

with the piecewise constant approximations $\left.\kappa_{h}^{\delta}\right|_{x_{k}^{\delta}\left(S_{i}\right)}(x):=\kappa\left(x_{k}, \frac{x_{k}^{\delta}\left(y_{i}\right)}{\delta}\right)$ for all $S_{i} \in$ $\mathcal{T}_{h}\left(Y_{k}^{\delta}\right)$ and $\left(\mu^{-1}\right)_{h}^{\delta}$ defined analogously. The local reconstructions $\left.\mathbf{R}_{j, 1}\left(\mathbf{u}_{H}\right) \in \mathbf{u}_{H}\right|_{Y_{j}^{\delta}}+$ $\widetilde{\mathbf{W}}_{h}^{1}\left(Y_{j}^{\delta}\right), \mathbf{R}_{j, 2}\left(\mathbf{u}_{H}\right)=\left.\mathbf{u}_{H}\left(x_{j}\right)\right|_{Y_{j}^{\delta}}+\nabla_{y} u_{h}$ with $u_{h} \in \widetilde{W}_{h}^{1}\left(Y_{j}^{\delta}\right)$ are defined as the solutions of the local cell problems

$$
\begin{aligned}
& \int_{Y_{j}^{\delta}}\left(\mu^{-1}\right)_{h}^{\delta}(x) \operatorname{curl} \mathbf{R}_{j, 1}\left(\mathbf{u}_{H}\right) \cdot \operatorname{curl} \boldsymbol{\psi}_{h}^{*}+\operatorname{div}\left(\mathbf{R}_{j, 1}\left(\mathbf{u}_{H}\right)-\mathbf{u}_{H}\right) \operatorname{div} \boldsymbol{\psi}_{h}^{*} d x=0 \\
& \left.\forall \boldsymbol{\psi}_{h} \in \widetilde{\mathbf{W}}_{h}^{1}\left(Y_{j}^{\delta}\right)\right), \\
& \int_{Y_{j}^{\delta}} \kappa_{h}^{\delta}(x) \mathbf{R}_{j, 2}\left(\mathbf{u}_{H}\right) \cdot \nabla \psi_{h}^{*} d x=0 \quad \forall \psi_{h} \in \widetilde{W}_{h}^{1}\left(Y_{j}^{\delta}\right) .
\end{aligned}
$$

Copyright $@$ by SIAM. Unauthorized reproduction of this article is prohibited. 
We now reformulate the reconstructions of the HMM solution triple to draw a parallel between them and the analytical correctors.

Remark 4.2 (role of the reconstructions). Let $\left(\mathbf{E}_{H}, \mathbf{R}_{1}\left(\mathbf{E}_{H}\right), \mathbf{R}_{2}\left(\mathbf{E}_{H}\right)\right.$ ) denote the HMM-approximation from Definition 4.1. Setting $\mathbf{K}_{j, 1}\left(\mathbf{E}_{H}\right)=\mathbf{R}_{j, 1}\left(\mathbf{E}_{H}\right)-\mathbf{E}_{H}$, we have $\mathbf{K}_{j, 1} \in \widetilde{\mathbf{W}}_{h}^{1}\left(Y_{j}^{\delta}\right)$. Furthermore, denote by $K_{j, 2}\left(\mathbf{E}_{H}\right) \in \widetilde{W}_{h}^{1}\left(Y_{j}^{\delta}\right)$ the function fulfilling $\nabla K_{j, 2}\left(\mathbf{E}_{H}\right)=\mathbf{R}_{j, 2}\left(\mathbf{E}_{H}\right)-\mathbf{E}_{H}\left(x_{j}\right)$. We then define the discrete fine-scale corrections $\mathbf{K}_{h, 1}\left(\mathbf{E}_{H}\right) \in S_{H}^{0}\left(\Omega ; \widetilde{\mathbf{W}}_{h}^{1}(Y)\right)$ and $K_{h, 2}\left(\mathbf{E}_{H}\right) \in S_{H}^{1}\left(\Omega ; \widetilde{W}_{h}^{1}(Y)\right)$ as

$$
\begin{aligned}
\left.\mathbf{K}_{h, 1}\left(\mathbf{E}_{H}\right)(x, y)\right|_{T_{j} \times Y} & :=\frac{1}{\delta} \mathbf{K}_{j, 1}\left(\mathbf{E}_{H}\right)(\delta y), \\
K_{h, 2}\left(\mathbf{E}_{H}\right)\left(x_{l}, y\right) & :=\frac{1}{\delta} K_{l, 2}\left(\mathbf{E}_{H}\right)(\delta y) \quad \forall x_{l} \in Q_{j}^{(2)} \forall T_{j} \in \mathcal{T}_{H},
\end{aligned}
$$

where the space of piecewise $p$-polynomial, $p \in \mathbb{N}_{0}$, (with respect to $x$ ) discrete functions is defined as

$$
\begin{gathered}
S_{H}^{p}\left(\Omega ; \widetilde{W}_{h}^{1}(Y)\right):=\left\{u_{h} \in L^{2}\left(\Omega, H_{\sharp, 0}^{1}(Y)\right)\left|u_{h}(\cdot, y)\right|_{T_{j}} \in \mathbb{P}^{p} \forall j \in J, y \in Y\right. \\
\text { and } \left.u_{h}(x, \cdot) \in \widetilde{W}_{h}^{1}(Y) \forall x \in \Omega\right\} .
\end{gathered}
$$

The discrete fine-scale corrections $\mathbf{K}_{h, 1}\left(\mathbf{E}_{H}\right), K_{h, 2}\left(\mathbf{E}_{H}\right)$ are discrete counterparts of the analytical correctors $\mathbf{K}_{1}$ and $K_{2}$ introduced in Theorem 3.2. The specific relation of both will be clear from Proposition 4.3 below. Therefore, these corrections (or equivalently the reconstructions) are an important part of the HMM-approximation. As discussed at the end of section 3, the correctors carry important information on the solution and cannot be neglected as higher order terms (in contrast to diffusion problems). In the form of the fine-scale corrections, the observation transfers to the numerical scheme and the discrete setting.

Having observed this correspondence, we can now reformulate the whole HMM to see that it is a direct discretization with numerical quadrature of the two-scale equation (3.1); see [38].

Proposition 4.3 (reformulation of the HMM). Define the piecewise constant approximations $\kappa_{h}$ on $\Omega \times Y$ by $\left.\kappa_{h}(x, y)\right|_{T_{j} \times S_{i}}:=\kappa\left(x_{j}, y_{i}\right)$ and $\mu_{h}^{-1}$ in the same way. Furthermore, let $\mathbf{K}_{h, 1}, K_{h, 2}$ be the discrete fine-scale corrections as defined in Remark 4.2. Then $\left(\mathbf{E}_{H}, \mathbf{K}_{h, 1}\left(\mathbf{E}_{H}\right), K_{h, 2}\left(\mathbf{E}_{H}\right)\right) \in \mathbf{V}_{H, 0}^{I} \times S_{H}^{0}\left(\Omega ; \widetilde{\mathbf{W}}_{h}^{1}(Y)\right) \times S_{H}^{1}\left(\Omega ; \widetilde{W}_{h}^{1}(Y)\right)$ is a solution of

$$
\begin{aligned}
& \mathcal{B}_{h}\left(\left(\mathbf{E}_{H}, \mathbf{K}_{h, 1}\left(\mathbf{E}_{H}\right), K_{h, 2}\left(\mathbf{E}_{H}\right)\right),\left(\boldsymbol{\psi}_{H}, \boldsymbol{\psi}_{h}, \psi_{h}\right)\right)=\int_{\Omega} \mathbf{f}(x) \cdot \boldsymbol{\psi}_{H}^{*}(x) d x \\
& \forall\left(\boldsymbol{\psi}_{H}, \boldsymbol{\psi}_{h}, \psi_{h}\right) \in \mathbf{V}_{H, 0}^{I} \times L^{2}\left(\Omega ; \widetilde{\mathbf{W}}_{h}^{1}(Y)\right) \times L^{2}\left(\Omega ; \widetilde{W}_{h}^{1}(Y)\right)
\end{aligned}
$$

with the discrete sesquilinear form $\mathcal{B}_{h}$ given as

$$
\begin{aligned}
& \mathcal{B}_{h}\left(\left(\mathbf{u}_{H}, \mathbf{u}_{h}, u_{h}\right),\left(\boldsymbol{\psi}_{H}, \boldsymbol{\psi}_{h}, \psi_{h}\right)\right) \\
& :=\int_{\Omega} \int_{Y} \mu_{h}^{-1}(x, y)\left(\operatorname{curl}_{\mathbf{u}_{H}}(x)+\operatorname{curl}_{y} \mathbf{u}_{h}(x, y)\right) \cdot\left(\operatorname{curl} \boldsymbol{\psi}_{H}^{*}(x)+\operatorname{curl}_{y} \boldsymbol{\psi}_{h}^{*}(x, y)\right) \\
& \quad+\operatorname{div}_{y} \mathbf{u}_{h}(x, y) \operatorname{div}_{y} \boldsymbol{\psi}_{h}^{*}(x, y) \\
& \quad-\kappa_{h}(x, y)\left(\mathbf{u}_{H}(x)+\nabla_{y} u_{h}(x, y)\right) \cdot\left(\boldsymbol{\psi}_{H}^{*}(x)+\nabla_{y} \psi_{h}^{*}(x, y)\right) d y d x .
\end{aligned}
$$

Copyright $@$ ㅇ by SIAM. Unauthorized reproduction of this article is prohibited. 
Proof. We treat the two terms of the sesquilinear form separately but with basically the same procedure. For the first term we see from the definition of the reconstruction $\mathbf{R}_{1}$ that for all $\boldsymbol{\psi}_{h} \in \widetilde{\mathbf{W}}_{h}^{1}\left(Y_{j}^{\delta}\right)$ ) it holds that

$$
\begin{gathered}
0=\int_{Y_{j}^{\delta}}\left(\mu^{-1}\right)_{h}^{\delta}(x) \operatorname{curl}_{x}\left(\mathbf{E}_{H}+\mathbf{R}_{j, 1}\left(\mathbf{E}_{H}\right)-\mathbf{E}_{H}\right)(x) \cdot \operatorname{curl}_{x} \boldsymbol{\psi}_{h}^{*}(x) \\
+\operatorname{div}_{x}\left(\mathbf{R}_{j, 1}\left(\mathbf{E}_{H}\right)-\mathbf{E}_{H}\right)(x) \operatorname{div}_{x} \boldsymbol{\psi}_{h}^{*}(x) d x .
\end{gathered}
$$

Using the transformation formula and writing $x=x_{j}^{\delta}(y)$, we derive

$$
\begin{aligned}
0=\delta^{3} \int_{Y} \mu^{-1}\left(x_{j}, \frac{x_{j}^{\delta}(y)}{\delta}\right)\left(\operatorname{curl}_{x} \mathbf{E}_{H}\left(x_{j}\right)+\operatorname{curl}_{x} \mathbf{K}_{j, 1}\left(\mathbf{E}_{H}\right)\left(x_{j}^{\delta}(y)\right)\right) \cdot \operatorname{curl}_{x} \boldsymbol{\psi}_{h}^{*}\left(x_{j}^{\delta}(y)\right) \\
+\operatorname{div}_{x} \mathbf{K}_{j, 1}\left(\mathbf{E}_{H}\right)\left(x_{j}^{\delta}(y)\right) \operatorname{div}_{x} \boldsymbol{\psi}_{h}^{*}\left(x_{j}^{\delta}(y)\right) d y
\end{aligned}
$$

since $\operatorname{curl}_{x} \mathbf{E}_{H}(x)$ is constant on each $T_{j}$. Using the definition of $\mathbf{K}_{h, 1}\left(\mathbf{E}_{H}\right)$ and defining $\widetilde{\boldsymbol{\psi}}_{h} \in \widetilde{\mathbf{W}}_{h}^{1}(Y)$ as $\widetilde{\boldsymbol{\psi}}_{h}(y)=\frac{1}{\delta} \boldsymbol{\psi}_{h}(\delta y)$, we get with the chain rule

$$
\begin{aligned}
0=\delta^{3} \int_{Y} \mu^{-1}\left(x_{j}, \frac{x_{j}^{\delta}(y)}{\delta}\right) \operatorname{curl}_{y} \widetilde{\boldsymbol{\psi}}_{h}^{*}\left(\frac{x_{j}^{\delta}(y)}{\delta}\right) \\
\cdot\left(\operatorname{curl}_{x} \mathbf{E}_{H}\left(x_{j}\right)+\operatorname{curl}_{y} \mathbf{K}_{h, 1}\left(\mathbf{E}_{H}\right)\left(x_{j}, \frac{x_{j}^{\delta}(y)}{\delta}\right)\right) \\
+\operatorname{div}_{y} \mathbf{K}_{h, 1}\left(\mathbf{E}_{H}\right)\left(x_{j}, \frac{x_{j}^{\delta}(y)}{\delta}\right) \operatorname{div}_{y} \widetilde{\boldsymbol{\psi}}_{h}^{*}\left(\frac{x_{j}^{\delta}(y)}{\delta}\right) d y .
\end{aligned}
$$

As the integrand is $Y$-periodic and $\frac{x_{j}^{\delta}(y)}{\delta}=y+\frac{x_{j}}{\delta}$, we finally obtain the Galerkin orthogonality

$$
\begin{gathered}
0=\delta^{3} \int_{Y} \mu_{h}^{-1}\left(x_{j}, y\right)\left(\operatorname{curl}_{x} \mathbf{E}_{H}\left(x_{j}\right)+\operatorname{curl}_{y} \mathbf{K}_{h, 1}\left(\mathbf{E}_{H}\right)\left(x_{j}, y\right)\right) \cdot \operatorname{curl}_{y} \widetilde{\boldsymbol{\psi}}_{h}^{*}(y) \\
+\operatorname{div}_{y} \mathbf{K}_{h, 1}\left(\mathbf{E}_{H}\right)\left(x_{j}, y\right) \operatorname{div}_{y} \widetilde{\boldsymbol{\psi}}_{h}^{*}(y) d y
\end{gathered}
$$

In the same way, we deduce

$$
\begin{aligned}
& \sum_{j \in J} \frac{\left|T_{j}\right|}{\delta^{3}} \int_{Y_{j}^{\delta}}\left(\mu^{-1}\right)_{h}^{\delta}(x) \operatorname{curl}_{x} \mathbf{R}_{1}\left(\mathbf{E}_{H}\right)(x) \cdot \operatorname{curl} \boldsymbol{\psi}_{H}^{*}(x) d x \\
&= \sum_{j \in J}\left|T_{j}\right| \int_{Y} \mu^{-1}\left(x_{j}, y\right) \operatorname{curl} \boldsymbol{\psi}_{H}^{*}\left(x_{j}\right) \cdot\left(\operatorname{curl} \mathbf{E}_{H}\left(x_{j}\right)+\operatorname{curl}_{y} \mathbf{K}_{h, 1}\left(\mathbf{E}_{H}\right)\left(x_{j}, y\right)\right) d y \\
&= \int_{\Omega} \int_{Y} \mu_{h}^{-1}(x, y)\left(\operatorname{curl} \mathbf{E}_{H}(x)+\operatorname{curl}_{y} \mathbf{K}_{h, 1}\left(\mathbf{E}_{H}\right)(x, y)\right) \cdot\left(\operatorname{curl} \boldsymbol{\psi}_{H}^{*}(x)+\operatorname{curl}_{y} \boldsymbol{\psi}_{h}^{*}(x, y)\right) \\
& \quad+\operatorname{div}_{y} \mathbf{K}_{h, 1}\left(\mathbf{E}_{H}\right)(x, y) \operatorname{div}_{y} \boldsymbol{\psi}_{h}^{*}(x, y) d y d x
\end{aligned}
$$

In the last equality we used that the given quadrature rule is exact for the integrands and we employed the Galerkin orthogonality. For the second term in the sesquilinear form, one can perform the same steps to reformulate the problem. For this term the computations are very similar to the diffusion problem discussed in [38, Lemma 3.5].

Copyright $@$ by SIAM. Unauthorized reproduction of this article is prohibited. 
Conclusion 4.4. Let us note that the result of Theorem 3.5 is still valid if we replace $\nabla K_{2}$ by $\delta^{-1} \nabla_{y} K_{2}$. This implies that we can approximate $\mathbf{E}_{\delta}$ in $\mathbf{H}$ (curl) by $\mathbf{E}(x)+\delta \mathbf{K}_{1}\left(x, \frac{x}{\delta}\right)+\nabla_{y} K_{2}\left(x, \frac{x}{\delta}\right)$. Consequently, exploiting Proposition 4.3, we see that our final HMM-approximation $\mathbf{E}_{\mathrm{HмM}}$ to $\mathbf{E}_{\delta}$ can be written as

$$
\mathbf{E}_{\mathrm{HMM}}(x):=\mathbf{E}_{H}(x)+\delta \mathbf{K}_{h, 1}\left(\mathbf{E}_{H}\right)\left(x, \frac{x}{\delta}\right)+\nabla_{y} K_{h, 2}\left(\mathbf{E}_{H}\right)\left(x, \frac{x}{\delta}\right) .
$$

5. A priori and a posteriori error analysis. Based on the reformulation of the HMM in Proposition 4.3, we will give the main a priori and a posteriori error estimates in Theorems 5.2, 5.3, 5.4, and 5.5. All error estimates will be derived in the "two-scale energy norm"

$$
\begin{aligned}
& \left\|\left(\mathbf{u}, \mathbf{u}_{1}, u_{2}\right)\right\|_{e(G \times R)} \\
& \quad:=\left\|\operatorname{curl} \mathbf{u}+\operatorname{curl}_{y} \mathbf{u}_{1}\right\|_{L^{2}(G \times R)}+\left\|\operatorname{div}_{y} \mathbf{u}_{1}\right\|_{L^{2}(G \times R)}+\left\|\mathbf{u}+\nabla_{y} u_{2}\right\|_{L^{2}(G \times R)}
\end{aligned}
$$

for $G \times R \subset \Omega \times Y$ an open subdomain. If the norm is to be taken over $\Omega \times Y$, we will just write $\|\cdot\|_{e}$. Let us furthermore define the error terms $e_{0}:=\mathbf{E}-\mathbf{E}_{H}$, $e_{1}:=\mathbf{K}_{1}-\mathbf{K}_{h, 1}\left(\mathbf{E}_{H}\right)$, and $e_{2}:=K_{2}-K_{h, 2}\left(\mathbf{E}_{H}\right)$. We will only estimate these errors and leave the modeling error $\mathbf{E}_{\delta}-\left(\mathbf{E}+\delta\left(\mathbf{K}_{1}(\cdot, \dot{\bar{\delta}})+\nabla K_{2}(\cdot, \dot{\bar{\delta}})\right)\right)$, introduced by homogenization, apart. Conclusion 4.4 together with Theorem 3.5 as well as the explicit rate (3.6) from [12] shows that we can neglect the modeling error if $\delta$ is sufficiently small. More explicitly, we have the following estimate for the total error:

$$
\left\|\mathbf{E}_{\delta}-\left(\mathbf{E}_{H}+\delta\left(\mathbf{K}_{h, 1}\left(\cdot, \frac{\dot{\delta}}{\delta}\right)+\nabla K_{h, 2}\left(\cdot, \frac{\dot{\delta}}{\delta}\right)\right)\right)\right\|_{\mathbf{H}(\operatorname{curl})} \leq C \delta^{1 / 2}+\left\|\left(e_{0}, e_{1}, e_{2}\right)\right\|_{e} .
$$

The numerical experiment in section 7 will also justify this concentration on the discretization error.

Assumption 5.1. On top of the periodicity of the coefficients, we also assume

$$
\mu^{-1}, \kappa \in W^{1, \infty}(\Omega \times Y),
$$

i.e., the coefficient functions are globally Lipschitz, and $\Omega$ is a convex domain. This assumption will be required for the a priori estimates (Theorems 5.2 and 5.3) but not for the a posteriori estimates (Theorems 5.4 and 5.5).

Theorem 5.2 (a priori estimate in the energy norm). Under Assumptions 2.1 and 5.1, the following a priori estimate for the error between the homogenized solution and the HMM-approximation, respectively, their correctors holds:

$$
\left\|\left(e_{0}, e_{1}, e_{2}\right)\right\|_{e} \leq C(H+h)\|\mathbf{f}\|_{L^{2}(\Omega)} \quad \text { with } C=C\left(\Omega, \kappa, \mu^{-1}\right) .
$$

TheORem 5.3 (a priori error estimate with dual problems). Under the same assumptions as in Theorem 5.2, the Helmholtz decomposition of the error between the continuous solution $\mathbf{E}$ and the HMM-approximation $\mathbf{E}_{H}$

$$
\mathbf{E}-\mathbf{E}_{H}=\nabla \theta+\mathbf{z} \quad \text { with } \theta \in H_{0}^{1}(\Omega), \mathbf{z} \perp \nabla H_{0}^{1}(\Omega)
$$

satisfies

$$
\|\theta\|_{L^{2}(\Omega)}+\|\mathbf{z}\|_{L^{2}(\Omega)} \leq C\left(H^{2}+h^{2}\right)\|\mathbf{f}\|_{L^{2}(\Omega)}+C \eta_{\text {approx }}\|\mathbf{f}\|_{L^{2}(\Omega)},
$$

where $\eta_{\text {approx }}=\max \left\{\left\|\mu^{-1}-\mu_{h}^{-1}\right\|_{L^{\infty}(\Omega \times Y)},\left\|\kappa-\kappa_{h}\right\|_{L^{\infty}(\Omega \times Y)}\right\}$ is a data approximation error arising from numerical quadrature. The constant $C$ only depends on the domain $\Omega$ and the coefficients $\mu^{-1}$ and $\kappa$ but not on the periodicity parameter $\delta$ or the mesh sizes.

Copyright $@$ by SIAM. Unauthorized reproduction of this article is prohibited. 
For diffusion problems, posed on $H^{1}(\Omega)$, the $L^{2}$-norm of the error converges with a quadratic rate. This better convergence is obtained by posing a dual problem and using the Aubin-Nitsche trick. The above theorem shows how the result can be transferred to problems in $\mathbf{H}$ (curl): On the gradient subspace, the $L^{2}$-norm is of the same order as the $\mathbf{H}$ (curl)-norm, so that only on the complement a better convergence is obtained (see [41, Remark after Theorem 49, p. 45]). Hence, the quadratic convergence here is (only) obtained in $H^{-1}(\Omega)$.

TheOrem 5.4 (a posteriori error estimate). Let $\mathbf{f}_{H}$ be any piecewise polynomial approximation of f. Under the Assumption 2.1 the error fulfills the following a posteriori error estimate:

$$
\begin{aligned}
\left\|\left(e_{0}, e_{1}, e_{2}\right)\right\|_{e} \leq & C\left(\sum_{j \in J} \eta_{j, 1}^{2}+\eta_{j, 2}^{2}\right)^{1 / 2}+C\left(\sum_{(j, l) \in \mathcal{E}\left(\mathcal{T}_{H}\right)} \eta_{j l, 1}^{2}+\eta_{j l, 2}^{2}\right)^{1 / 2} \\
& +C\left(\sum_{j \in J} \sum_{(k, m) \in \mathcal{E}\left(\mathcal{T}_{h}\right)} \eta_{j, k m, 1}^{2}+\eta_{j, k m, 2}^{2}\right)^{1 / 2} \\
& +C\left(\sum_{j \in J} \zeta_{j}^{2}\right)^{1 / 2}+C\left(\sum_{j \in J} \sum_{k \in I} \zeta_{j k}^{2}\right)^{1 / 2}
\end{aligned}
$$

where the constants do not depend on the mesh sizes and the periodicity parameter $\delta$. The local indicators are defined as

$$
\begin{aligned}
& \eta_{j, 1}:=H_{j}\left\|\mathbf{f}_{H}+\int_{Y} \kappa_{h}(\cdot, y)\left(\mathbf{E}_{H}+\nabla_{y} K_{h, 2}(\cdot, y)\right) d y\right\|_{L^{2}\left(T_{j}\right)}, \\
& \eta_{j, 2}:=H_{j}\left\|\operatorname{div}_{x}\left(\int_{Y} \kappa_{h}(\cdot, y)\left(\mathbf{E}_{H}+\nabla_{y} K_{h, 2}(\cdot, y)\right) d y\right)\right\|_{L^{2}\left(T_{j}\right)}, \\
& \eta_{j l, 1}:=H_{j l}^{1 / 2}\left\|\left[\int_{Y} \mu_{h}^{-1}(\cdot, y)\left(\operatorname{curl} \mathbf{E}_{H}+\operatorname{curl}_{y} \mathbf{K}_{h, 1}(\cdot, y)\right) \times \mathbf{n} d y\right]_{F_{j l}}\right\|_{L^{2}\left(F_{j l}\right)}, \\
& \eta_{j l, 2}::=H_{j l}^{1 / 2}\left\|\left[\int_{Y} \kappa_{h}(\cdot, y)\left(\mathbf{E}_{H}+\nabla_{y} K_{h, 2}(\cdot, y)\right) \cdot \mathbf{n} d y\right]\right\|_{F_{j l}} \|_{L^{2}\left(F_{j l}\right)}, \\
& \eta_{j, k m, 1}:=h_{k m}^{1 / 2}\left\|\left[\mu_{h}^{-1}\left(\operatorname{curl} \mathbf{E}_{H}+\operatorname{curl}_{y} \mathbf{K}_{h, 1}\right) \times \mathbf{n}+\operatorname{div} \mathbf{K}_{h, 1} \mathbf{n}\right]_{\tilde{F}_{k m}}\right\|_{L^{2}\left(T_{j} \times \tilde{F}_{k m}\right)}, \\
& \eta_{j, k m, 2}:=h_{k m}^{1 / 2}\left\|\left[\kappa_{h}\left(\mathbf{E}_{H}+\nabla_{y} K_{h, 2}\right) \cdot \mathbf{n}\right]_{\tilde{F}_{k m}}\right\|_{L^{2}\left(T_{j} \times \tilde{F}_{k m}\right)}, \\
& \zeta_{j}:=H_{j}\left\|\mathbf{f}_{H}-\mathbf{f}\right\|_{L^{2}\left(T_{j}\right)}, \\
& \zeta_{j k}:=\left\|\left(\mu_{h}^{-1}-\mu^{-1}\right)\left(\operatorname{curl} \mathbf{E}_{H}+\operatorname{curl}_{y} \mathbf{K}_{h, 1}\right)\right\|_{L^{2}\left(T_{j} \times S_{k)}\right.} \\
&+\left\|\left(\kappa_{h}-\kappa\right)\left(\mathbf{E}_{H}+\nabla_{y} K_{h, 2}\right)\right\|_{L^{2}\left(T_{j} \times S_{k}\right)} .
\end{aligned}
$$

Here and in the following, $[\cdot]_{F}$ denotes the jump across the face $F$.

The error indicators can be split into two groups: $\zeta_{j}$ and $\zeta_{j k}$ are data approximation errors, which come from the use of numerical quadrature. The error indicators denoted by $\eta$ are different contributions to the discretization error: $\eta_{j, 1}$ is the element residual on the macroscale, $\eta_{j l}$ are the jump residuals on the macroscale (in normal and tangential direction), and $\eta_{j, k m}$ are the jump residuals on the microscale. $\eta_{j, 2}$ 
indicates how well the deduced equation $\operatorname{div}_{x}\left(\kappa \mathbf{E}_{0}\right)=0$ is fulfilled in the discrete case. Here, the assumption $\operatorname{div} \mathbf{f}=0$ (in the weak sense) has an effect on the error estimator: If we just assume $\mathbf{f} \in \mathbf{H}(\operatorname{div})$, the deduced equation is $\operatorname{div}_{x}\left(\kappa \mathbf{E}_{0}+\mathbf{f}\right)=0$, and thus we have additional terms $\operatorname{div}_{x} \mathbf{f}_{H}$ in $\eta_{j, 2}$ and $\mathbf{f}_{H} \cdot \mathbf{n}$ in $\eta_{j l, 2}$ for the polynomial approximation $\mathbf{f}_{H}$ of $\mathbf{f}$. Furthermore, in the data approximation error $\zeta_{j}$ we then have to take the $\mathbf{H}\left(\operatorname{div}, T_{j}\right)$-norm.

TheOREm 5.5 (lower bound on the error). With the same notation and under the same assumptions as in Theorem 5.4, the following local bounds on the error hold:

$$
\begin{aligned}
& \eta_{j, 1} \leq C\left(\left\|\left(e_{0}, e_{1}, e_{2}\right)\right\|_{e\left(T_{j} \times Y\right)}+\zeta_{j}+\left(\sum_{k} \zeta_{j k}^{2}\right)^{1 / 2}\right), \\
& \eta_{j, 2} \leq C\left(\left\|\left(e_{0}, e_{1}, e_{2}\right)\right\|_{e\left(T_{j} \times Y\right)}+\left(\sum_{k} \zeta_{j k}^{2}\right)^{1 / 2}\right), \\
& \eta_{j l, 1} \leq C\left(\left\|\left(e_{0}, e_{1}, e_{2}\right)\right\|_{e\left(\hat{\omega}_{F_{j l}} \times Y\right)}+\left(\zeta_{j}^{2}+\zeta_{l}^{2}\right)^{1 / 2}+\left(\sum_{k} \zeta_{j k}^{2}+\zeta_{l k}^{2}\right)^{1 / 2}\right), \\
& \eta_{j l, 2} \leq C\left(\left\|\left(e_{0}, e_{1}, e_{2}\right)\right\|_{e\left(\hat{\omega}_{F_{j l}} \times Y\right)}+\left(\sum_{k} \zeta_{j k}^{2}+\zeta_{l k}^{2}\right)^{1 / 2}\right), \\
& \eta_{j, k m, \nu} \leq C\left(\left\|\left(e_{0}, e_{1}, e_{2}\right)\right\|_{e\left(T_{j} \times \hat{\omega}_{\tilde{F}_{k m}}\right)}+\left(\zeta_{j k}^{2}+\zeta_{j m}^{2}\right)^{1 / 2}\right), \quad \nu=1,2 .
\end{aligned}
$$

Thus, we have the global estimate

$$
\begin{aligned}
& \left(\sum_{j \in J} \eta_{j, 1}^{2}+\eta_{j, 2}^{2}\right)^{1 / 2}+\left(\sum_{(j, l) \in \mathcal{E}\left(\mathcal{T}_{H}\right)} \eta_{j l, 1}^{2}+\eta_{j l, 2}^{2}\right)^{1 / 2} \\
& \quad+\left(\sum_{j \in J} \sum_{(k, m) \in \mathcal{E}\left(\mathcal{T}_{h}\right)} \eta_{j, k m, 1}^{2}+\eta_{j, k m, 2}^{2}\right)^{1 / 2} \\
& \quad \leq C\left(\left\|\left(e_{0}, e_{1}, e_{2}\right)\right\|_{e}+\left(\sum_{j \in J} \zeta_{j}^{2}\right)^{1 / 2}+\left(\sum_{j \in J} \sum_{k \in I} \zeta_{j k}^{2}\right)^{1 / 2}\right) .
\end{aligned}
$$

Theorems 5.4 and 5.5 together show that the local error indicators are reliable and efficient with respect to the two-scale homogenized problem from Theorem 3.2. Up to data approximation errors, the error and the indicators converge with the same rate with respect to the mesh parameters $H$ and $h$. Thus, the indicators can be used for adaptive algorithms, e.g., for mesh refinement, both on the coarse and fine scale.

6. Proofs of the main results. In this section, the essential proofs of the homogenization result (namely, the two-scale equation) and the error estimates for the HMM will be given.

Proof of Theorem 3.2. As solutions to (2.2) are uniformly bounded in $\mathbf{H}(\mathrm{curl})$, we have by Proposition 3.1 that, along a subsequence, $\mathbf{E}_{\delta} \stackrel{2}{\rightarrow} \mathbf{E}_{0}$ in $\left(L^{2}(\Omega \times Y)\right)^{3}$, $\operatorname{curl} \mathbf{E}_{\delta} \stackrel{2}{\rightarrow} \operatorname{curl} \mathbf{E}+\operatorname{curl}_{y} \mathbf{K}_{1}$, and $\mathbf{E}_{0}=\mathbf{E}+\nabla_{y} K_{2}$, where $\mathbf{E}=\int_{Y} \mathbf{E}_{0}(\cdot, y) d y$ is the 
weak limit of $\mathbf{E}_{\delta}$ in $\mathbf{H}$ (curl). We insert $\boldsymbol{\psi}(x)=\mathbf{w}(x)+\delta \mathbf{w}_{1}\left(x, \frac{x}{\delta}\right)$ with arbitrary $\mathbf{w} \in$ $\left(C_{0}^{\infty}(\Omega)\right)^{3}, \mathbf{w}_{1} \in C_{0}^{\infty}\left(\Omega ;\left(C_{\sharp}^{\infty}(Y)\right)^{3}\right)$ as a test function in $(2.2)$. Because of Assumption 2.1 on the parameters we can apply two-scale convergence to each of the terms in (2.2) and thereby obtain

$$
\begin{aligned}
& \int_{\Omega} \int_{Y} \mu^{-1}(x, y)\left(\operatorname{curl} \mathbf{E}(x)+\operatorname{curl}_{y} \mathbf{K}_{1}(x, y)\right) \cdot\left(\operatorname{curl} \mathbf{w}^{*}(x)+\operatorname{curl}_{y} \mathbf{w}_{1}^{*}(x, y)\right) \\
& \quad-\kappa(x, y)\left(\mathbf{E}(x)+\nabla_{y} K_{2}(x, y)\right) \cdot \mathbf{w}^{*}(x) d y d x \\
& \quad=\int_{\Omega} \mathbf{f}(x) \cdot \mathbf{w}^{*}(x) d x .
\end{aligned}
$$

By density this also holds for test functions in $\mathbf{H}_{0}(\operatorname{curl}) \times L^{2}\left(\Omega ; \mathbf{H}_{\sharp, 0}^{1}(Y)\right)$. Furthermore, we can deduce from (2.2) with $\operatorname{div} \mathbf{f}=0$ that it holds that

$$
\delta \int_{\Omega} \kappa_{\delta}(x) \mathbf{E}_{\delta}(x) \cdot \nabla \psi^{*}(x) d x=0 \quad \forall \psi \in H_{0}^{1}(\Omega) .
$$

Choosing $\psi\left(x, \frac{x}{\delta}\right) \in C_{0}^{\infty}\left(\Omega ; C_{\sharp}^{\infty}(Y)\right)$, we obtain with two-scale convergence

$$
\int_{\Omega} \int_{Y} \kappa(x, y)\left(\mathbf{E}(x)+\nabla_{y} K_{2}(x, y)\right) \cdot \nabla_{y} \psi^{*}(x, y) d y d x=0 .
$$

Inserting this into (6.1), we get (3.1) except for the divergence term, but with the additional constraint $\operatorname{div}_{y} \mathbf{K}_{1}=0$.

As discussed in section 3, we can apply divergence-regularization in this case to obtain an equivalent problem. Looking at the method, we directly see that (3.1) is simply the regularization of (6.1).

So far we have shown (3.1) just for a subsequence. To obtain the result for the whole sequence, we show the uniqueness of a solution to (3.1) with the help of the Lax-Milgram-Babuška theorem. We consider the Hilbert space

$$
\mathbf{H}:=\mathbf{H}_{0}(\operatorname{curl}) \times L^{2}\left(\Omega ; \mathbf{H}_{\sharp, 0}^{1}(Y)\right) \times L^{2}\left(\Omega ; H_{\sharp, 0}^{1}(Y)\right)
$$

with its natural norm

$$
\left\|\left(\mathbf{u}, \mathbf{u}_{1}, u_{2}\right)\right\|_{\mathbf{H}}^{2}=\|\mathbf{u}\|_{\mathbf{H}(\text { curl })}^{2}+\left\|\mathbf{u}_{1}\right\|_{L^{2}\left(\Omega ; \mathbf{H}^{1}(Y)\right)}^{2}+\left\|u_{2}\right\|_{L^{2}\left(\Omega ; H^{1}(Y)\right)}^{2} .
$$

Clearly, the right-hand side is in the dual space of $\mathbf{H}$ and the left-hand side defines a continuous sesquilinear form $\mathcal{B}$. With the same computations as for the existence of a weak solution, one can also show that $\mathcal{B}$ is coercive with respect to the energy norm

$$
\left\|\operatorname{curl} \mathbf{u}+\operatorname{curl}_{y} \mathbf{u}_{1}\right\|_{L^{2}(\Omega \times Y)}^{2}+\left\|\operatorname{div}_{y} \mathbf{u}_{1}\right\|_{L^{2}(\Omega \times Y)}^{2}+\left\|\mathbf{u}+\nabla_{y} u_{2}\right\|_{L^{2}(\Omega \times Y)}^{2} .
$$

It remains to show the equivalence of the energy and the natural norm. It holds that

$$
\begin{aligned}
\left\|\operatorname{curl} \mathbf{u}+\operatorname{curl}_{y} \mathbf{u}_{1}\right\|_{L^{2}(\Omega \times Y)}^{2} & =\int_{\Omega} \int_{Y}|\operatorname{curl} \mathbf{u}|^{2}+\left|\operatorname{curl}_{y} \mathbf{u}_{1}\right|^{2}+2 \operatorname{Re}\left(\operatorname{curl}_{y} \mathbf{u}_{1}(x, y) \cdot \operatorname{curl} \mathbf{u}^{*}(x)\right) d y d x \\
= & \|\operatorname{curl} \mathbf{u}\|_{L^{2}(\Omega)}^{2}+\left\|\operatorname{curl}_{y} \mathbf{u}_{1}\right\|_{L^{2}(\Omega \times Y)}^{2} \\
& -2 \operatorname{Re} \underbrace{\left(\int_{\Omega} \int_{\partial Y}\left(\mathbf{u}_{1}(x, y) \times \mathbf{n}\right) \cdot \mathbf{u}^{*}(x) d \sigma d x\right)}_{=0, \text { periodicity of } \mathbf{u}_{1}} \\
= & \|\operatorname{curl} \mathbf{u}\|_{L^{2}(\Omega)}^{2}+\| \text { curl } \mathbf{u}_{1} \|_{L^{2}(\Omega \times Y)}^{2} .
\end{aligned}
$$

Copyright $@$ by SIAM. Unauthorized reproduction of this article is prohibited. 
With integration by parts and the Poincaré inequality we see that $\left\|\operatorname{curl}_{y} \mathbf{u}_{1}\right\|_{L^{2}}+$ $\left\|\operatorname{div}_{y} \mathbf{u}_{1}\right\|_{L^{2}}$ is equivalent to the full $H^{1}(Y)$-norm. The equivalence for the last part can be obtained similarly.

For the proofs of the errors estimates, we use the variational formulations and the related sesquilinear forms $\mathcal{B}$ and $\mathcal{B}_{h}$ from Theorem 3.2 and Proposition 4.3 and the notation for the residual given below.

Notation 6.1 (residual). We define the residual

$$
\begin{aligned}
& \operatorname{Res}_{h}: \mathbf{H}_{0}(\operatorname{curl}, \Omega) \times L^{2}\left(\Omega ; \mathbf{H}_{\sharp, 0}^{1}(Y)\right) \times L^{2}\left(\Omega ; H_{\sharp, 0}^{1}(Y)\right) \\
& \rightarrow \mathbf{H}_{0}(\operatorname{curl})^{\prime} \times L^{2}\left(\Omega ;\left(\mathbf{H}_{\sharp, 0}^{-1}(Y)\right)^{3}\right) \times L^{2}\left(\Omega ; H_{\sharp, 0}^{-1}(Y)\right) \\
& \text { as } \quad\left\langle\operatorname{Res}_{h}\left(\mathbf{u}, \mathbf{u}_{1}, u_{2}\right),\left(\boldsymbol{\psi}, \boldsymbol{\psi}_{1}, \psi_{2}\right)\right\rangle:=\mathcal{B}_{h}\left(\left(\mathbf{u}, \mathbf{u}_{1}, u_{2}\right),\left(\boldsymbol{\psi}, \boldsymbol{\psi}_{1}, \psi_{2}\right)\right)-(\mathbf{f}, \boldsymbol{\psi})
\end{aligned}
$$

with the discrete sequilinear form $\mathcal{B}_{h}$ defined in Proposition 4.3.

In the following, we will write $\mathbf{K}_{h, 1}$ instead of $\mathbf{K}_{h, 1}\left(\mathbf{E}_{H}\right)$, and $K_{h, 2}$ instead of $K_{h, 2}\left(\mathbf{E}_{H}\right)$. If it is clear on which variables $(x, y)$ functions depend, we will omit those variables for the sake of readability. $C$ denotes a generic constant, independent of the mesh sizes and $\delta$.

6.1. Proofs of the a priori estimates. The a priori estimates are based on the Céa lemma, dual problems, and interpolation operators. Assumption 5.1 implies higher regularity of the two-scale solution in the following way: With [16] and [41] we deduce $\mathbf{E} \in \mathbf{H}^{1}$ (curl) (defined in (2.1)) and $\mathbf{K}_{1} \in L^{2}\left(\Omega ; \mathbf{H}^{2}(Y)\right.$ ), and with Friedrich's theorem we obtain $K_{2} \in L^{2}\left(\Omega ; H^{2}(Y)\right)$, cf. [27, Folgerung 3.2.12]. The corresponding norms of the two-scale solution can be bounded by the right-hand side $\mathbf{f}$. Therefore, we can apply the following nodal interpolation operators.

Lemma 6.2 (nodal interpolation operators). Denote by $\tilde{I}_{h}^{L}: L^{2}\left(\Omega ; C^{0}(\bar{Y})\right) \cap$ $L^{2}\left(\Omega ; H_{\sharp, 0}^{1}(Y)\right) \rightarrow L^{2}\left(\Omega ; \widetilde{W}_{h}^{1}(Y)\right)$ the Lagrange interpolation operator, adapted to zero average. It fulfills the estimate

$$
\left\|\tilde{I}_{h}^{L}(v)-v\right\|_{L^{2}(\Omega \times Y)}+h\left|\tilde{I}_{h}^{L}(v)-v\right|_{L^{2}\left(\Omega, H^{1}(Y)\right)} \leq C h^{2}|v|_{L^{2}\left(\Omega, H^{2}(Y)\right)} .
$$

For vector functions the interpolation operator is defined componentwise and fulfills the same estimate. Denote by $I_{H}^{E}: \mathbf{H}^{1}(\mathrm{curl}) \rightarrow \mathbf{V}_{H, 0}^{I}$ the nodal interpolation operator for the Nédélec elements, where the space $\mathbf{H}^{1}$ (curl) is defined in (2.1). It fulfills the estimate

$$
\left\|\mathbf{u}-I_{H}^{E}(\mathbf{u})\right\|_{\mathbf{H}\left(\operatorname{curl}, T_{j}\right)} \leq C H_{j}\|\mathbf{u}\|_{\mathbf{H}^{1}\left(\operatorname{curl}, T_{j}\right)} .
$$

Proof. For the definition of $\tilde{I}_{h}^{L}$ and a proof of the estimate we refer the reader to [27]. For the definition of $I_{H}^{E}$ and the proof of the corresponding estimate we refer to [37, Theorem 5.41].

Proof of Theorem 5.2. Denote by $\widetilde{\mathbf{E}}_{H} \in \mathbf{V}_{H, 0}^{I}, \widetilde{\mathbf{E}}_{h} \in L^{2}\left(\Omega ; \widetilde{\mathbf{W}}_{h}^{1}(Y)\right)$, and $\widetilde{E}_{h} \in$ $L^{2}\left(\Omega ; \widetilde{W}_{h}^{1}(Y)\right)$ the unique solution of

$$
\begin{aligned}
& \mathcal{B}\left(\left(\widetilde{\mathbf{E}}_{H}, \widetilde{\mathbf{E}}_{h}, \widetilde{E}_{h}\right),\left(\boldsymbol{\psi}_{H}, \boldsymbol{\psi}_{h}, \psi_{h}\right)\right)=\left(\mathbf{f}, \boldsymbol{\psi}_{H}\right) \\
& \quad \forall \boldsymbol{\psi}_{H} \in \mathbf{V}_{H, 0}^{I}, \boldsymbol{\psi}_{h} \in L^{2}\left(\Omega ; \widetilde{\mathbf{W}}_{h}^{1}(Y)\right), \psi_{h} \in L^{2}\left(\Omega ; \widetilde{W}_{h}^{1}(Y)\right) .
\end{aligned}
$$

Copyright $@$ by SIAM. Unauthorized reproduction of this article is prohibited. 
With Céa's Lemma and the interpolation estimates of Lemma 6.2 we now derive

$$
\begin{aligned}
& \left\|\left(\mathbf{E}-\widetilde{\mathbf{E}}_{H}, \mathbf{K}_{1}-\widetilde{\mathbf{E}}_{h}, K_{2}-\widetilde{E}_{h}\right)\right\|_{e} \\
& \quad \leq C\left(\left\|\mathbf{E}-I_{H}^{E}(\mathbf{E})\right\|_{\mathbf{H}(\operatorname{curl})}+\left|\mathbf{K}_{1}-\tilde{I}_{h}^{L}\left(\mathbf{K}_{1}\right)\right|_{L^{2}\left(\Omega ; \mathbf{H}^{1}(Y)\right)}+\left|K_{2}-\tilde{I}_{h}^{L}\left(K_{2}\right)\right|_{L^{2}\left(\Omega ; H^{1}(Y)\right)}\right) \\
& \quad \leq C\left(H\|\mathbf{E}\|_{\mathbf{H}^{1}(\operatorname{curl})}+h\left|\mathbf{K}_{1}\right|_{L^{2}\left(\Omega ; \mathbf{H}^{2}(Y)\right)}+h\left|K_{2}\right|_{L^{2}\left(\Omega ; H^{2}(Y)\right)}\right) \\
& \quad \leq C(H+h)\|\mathbf{f}\|_{L^{2}(\Omega)},
\end{aligned}
$$

where in the last inequality we used regularity and stability results for the analytic solution. (Note that because of our assumptions on the parameters and on $\Omega$ the two-scale solution admits $H^{2}(Y)$ and $\mathbf{H}^{1}$ (curl) regularity as discussed above.) Furthermore, because of the definition of $\left(\widetilde{\mathbf{E}}_{H}, \widetilde{\mathbf{E}}_{h}, \widetilde{E}_{h}\right)$ it holds that

$$
\begin{aligned}
& \left\|\left(\widetilde{\mathbf{E}}_{H}-\mathbf{E}_{H}, \widetilde{\mathbf{E}}_{h}-\mathbf{K}_{h, 1}\left(\mathbf{E}_{H}\right), \widetilde{E}_{h}-K_{h, 2}\left(\mathbf{E}_{H}\right)\right)\right\|_{e}^{2} \\
& \leq C \mid\left(\mathcal{B}_{h}-\mathcal{B}\right)\left(\left(\mathbf{E}_{H}, \mathbf{K}_{h, 1}\left(\mathbf{E}_{H}\right), K_{h, 2}\left(\mathbf{E}_{H}\right)\right),\right. \\
& \left.\quad\left(\widetilde{\mathbf{E}}_{H}-\mathbf{E}_{H}, \widetilde{\mathbf{E}}_{h}-\mathbf{K}_{h, 1}\left(\mathbf{E}_{H}\right), \widetilde{E}_{h}-K_{h, 2}\left(\mathbf{E}_{H}\right)\right)\right) \mid \\
& \leq C \max \left\{\left\|\mu_{h}^{-1}-\mu^{-1}\right\|_{L^{\infty}(\Omega \times Y},\left\|\kappa_{h}-\kappa\right\|_{L^{\infty}(\Omega \times Y)}\right\} \\
& \quad\left\|\left(\mathbf{E}_{H}, \mathbf{K}_{h, 1}\left(\mathbf{E}_{H}\right), K_{h, 2}\left(\mathbf{E}_{H}\right)\right)\right\|_{e} \\
& \quad\left\|\left(\widetilde{\mathbf{E}}_{H}-\mathbf{E}_{H}, \widetilde{\mathbf{E}}_{h}-\mathbf{K}_{h, 1}\left(\mathbf{E}_{H}\right), \widetilde{E}_{h}-K_{h, 2}\left(\mathbf{E}_{H}\right)\right)\right\|_{e}
\end{aligned}
$$

From the Lipschitz continuity (with constant $L$ ) it follows that

$$
\left\|\kappa_{h}-\kappa\right\|_{L^{\infty}(\Omega \times Y)} \leq L \sup _{(x, y) \in \Omega \times Y}\left|\left(x_{i}, y_{k}\right)-(x, y)\right| \leq L(H+h),
$$

and the same estimate also applies to $\left\|\mu_{h}^{-1}-\mu^{-1}\right\|_{L^{\infty}(\Omega \times Y)}$. Together with a stability estimate for the HMM approximation this yields

$$
\left\|\left(\widetilde{\mathbf{E}}_{H}-\mathbf{E}_{H}, \widetilde{\mathbf{E}}_{h}-\mathbf{K}_{h, 1}\left(\mathbf{E}_{H}\right), \widetilde{E}_{h}-K_{h, 2}\left(\mathbf{E}_{H}\right)\right)\right\|_{e} \leq C(H+h)\|\mathbf{f}\|_{L^{2}(\Omega)} .
$$

Splitting the total error $\mathbf{E}-\mathbf{E}_{H}$ into the contributions $\mathbf{E}-\widetilde{\mathbf{E}}_{H}$ and $\widetilde{\mathbf{E}}_{H}-\mathbf{E}_{H}$ and using the two estimates, we obtain the assertion.

Proof of Theorem 5.3. As the terms in the Helmholtz decomposition are orthogonal with respect to the $L^{2}$-scalar product, we have

$$
\|\nabla \theta\|_{L^{2}} \leq\|\nabla \theta\|_{L^{2}}+\|\mathbf{z}\|_{L^{2}}=\left\|\mathbf{E}-\mathbf{E}_{H}\right\|_{L^{2}} .
$$

To estimate $\mathbf{z}$, let $\left(\mathbf{w}, \mathbf{w}_{1}, w_{2}\right)$ be the solution of the dual problem

$$
\mathcal{B}\left(\left(\boldsymbol{\psi}, \boldsymbol{\psi}_{1}, \psi_{2}\right),\left(\mathbf{w}, \mathbf{w}_{1}, w_{2}\right)\right)=(\mathbf{z}, \boldsymbol{\psi})_{L^{2}} \quad \forall\left(\boldsymbol{\psi}, \boldsymbol{\psi}_{1}, \psi_{2}\right)
$$

and $\left(\mathbf{w}_{H}, \mathbf{w}_{h}, w_{h}\right)$ the solution of the corresponding discrete dual problem

$$
\mathcal{B}_{h}\left(\left(\boldsymbol{\psi}_{H}, \boldsymbol{\psi}_{h}, \psi_{h}\right),\left(\mathbf{w}_{H}, \mathbf{w}_{h}, w_{h}\right)\right)=\left(\mathbf{z}, \boldsymbol{\psi}_{H}\right)_{L^{2}} \quad \forall\left(\boldsymbol{\psi}_{H}, \boldsymbol{\psi}_{h}, \psi_{h}\right) .
$$

The analytical and discrete spaces are the same as in the problems for $\mathbf{E}$ and $\mathbf{E}_{H}$ and therefore are not given again here. Because of $(\nabla \theta, \mathbf{z})=0$ it holds that $\|\mathbf{z}\|_{L^{2}}^{2}=$ $\left(\mathbf{z}, \mathbf{E}-\mathbf{E}_{H}\right)$. Thus, it follows that

$$
\|\mathbf{z}\|_{L^{2}}^{2}=\left(\mathbf{z}, \mathbf{E}-\mathbf{E}_{H}\right)_{L^{2}}=\mathcal{B}\left(\left(e_{0}, e_{1}, e_{2}\right),\left(\mathbf{w}, \mathbf{w}_{1}, w_{2}\right)\right)
$$


Using the definition of $\mathbf{E}$ as an exact solution and of $\mathbf{E}_{H}$ as the HMM-approximation, we deduce

$$
\begin{aligned}
\|\mathbf{z}\|_{L^{2}}^{2}= & \mathcal{B}\left(\left(e_{0}, e_{1}, e_{2}\right),\left(\mathbf{w}, \mathbf{w}_{1}, w_{2}\right)\right)-\mathcal{B}\left(\left(\mathbf{E}, \mathbf{K}_{1}, K_{2}\right),\left(\mathbf{w}_{H}, \mathbf{w}_{h}, w_{h}\right)\right) \\
& +\mathcal{B}_{h}\left(\left(\mathbf{E}_{H}, \mathbf{K}_{h, 1}\left(\mathbf{E}_{H}\right), K_{h, 2}\left(\mathbf{E}_{H}\right)\right),\left(\mathbf{w}_{H}, \mathbf{w}_{h}, w_{h}\right)\right) \\
= & \mathcal{B}\left(\left(e_{0}, e_{1}, e_{2}\right),\left(\mathbf{w}-\mathbf{w}_{H}, \mathbf{w}_{1}-\mathbf{w}_{h}, w_{2}-w_{h}\right)\right) \\
& +\left(\mathcal{B}_{h}-\mathcal{B}\right)\left(\left(\mathbf{E}_{H}, \mathbf{K}_{h, 1}\left(\mathbf{E}_{H}\right), K_{h, 2}\left(\mathbf{E}_{H}\right)\right),\left(\mathbf{w}_{H}, \mathbf{w}_{h}, w_{h}\right)\right) \\
\leq & C\left\|\left(e_{0}, e_{1}, e_{2}\right)\right\|_{e}\left\|\left(\mathbf{w}-\mathbf{w}_{H}, \mathbf{w}_{1}-\mathbf{w}_{h}, w_{2}-w_{h}\right)\right\|_{e} \\
& +C \max \left\{\left\|\mu^{-1}-\mu_{h}^{-1}\right\|_{L^{\infty}(\Omega \times Y},\left\|\kappa-\kappa_{h}\right\|_{L^{\infty}(\Omega \times Y)}\right\} \\
& \left\|\left(\mathbf{E}_{H}, \mathbf{K}_{h, 1}\left(\mathbf{E}_{H}\right), K_{h, 2}\left(\mathbf{E}_{H}\right)\right)\right\|_{e}\left\|\left(\mathbf{w}_{H}, \mathbf{w}_{h}, w_{h}\right)\right\|_{e} .
\end{aligned}
$$

According to Theorem 5.2, it holds that

$$
\left\|\left(\mathbf{w}-\mathbf{w}_{H}, \mathbf{w}_{1}-\mathbf{w}_{h}, w_{2}-w_{h}\right)\right\|_{e} \leq C(H+h)\|\mathbf{z}\|_{L^{2}} .
$$

Hence, together with stability estimates for $\mathbf{E}_{H}$ and $\mathbf{w}_{H}$ it follows that

$$
\|\mathbf{z}\|_{L^{2}}^{2} \leq C(H+h)\|\mathbf{z}\|_{L^{2}}\left\|\left(e_{0}, e_{1}, e_{2}\right)\right\|_{e}+C \eta_{\text {approx }}\|\mathbf{z}\|_{L^{2}}\|\mathbf{f}\|_{L^{2}}
$$

To estimate $\theta$, we pose another dual problem: Find $\hat{w} \in H_{0}^{1}(\Omega)$ and $\hat{w}_{2} \in$ $L^{2}\left(\Omega ; H_{\sharp, 0}^{1}(Y)\right)$ such that

$$
\mathcal{A}\left(\left(\hat{\psi}, \hat{\psi}_{2}\right),\left(\hat{w}, \hat{w}_{2}\right)\right)=(\theta, \hat{\psi})_{L^{2}} \quad \forall\left(\hat{\psi}, \hat{\psi}_{2}\right) \in H_{0}^{1}(\Omega) \times L^{2}\left(\Omega ; H_{\sharp, 0}^{1}(Y)\right)
$$

with

$$
\begin{aligned}
& \mathcal{A}\left(\left(\hat{\psi}, \hat{\psi}_{2}\right),\left(\hat{u}, \hat{u}_{2}\right)\right) \\
& \quad:=-\int_{\Omega} \int_{Y} \kappa(x, y)\left(\nabla \hat{\psi}(x)+\nabla_{y} \hat{\psi}_{2}(x, y)\right) \cdot\left(\nabla \hat{u}^{*}(x)+\nabla_{y} \hat{u}_{2}^{*}(x, y)\right) d y d x .
\end{aligned}
$$

Again, let us denote by $\left(\hat{w}_{H}, \hat{w}_{h}\right) \in W_{H}^{1}(\Omega) \times L^{2}\left(\Omega ; \widetilde{W}_{h}^{1}(Y)\right)$ the solution of the corresponding discrete dual problem. This dual problem is related to our original problem by the equation

$$
\mathcal{A}\left(\left(\hat{\psi}, \hat{\psi}_{2}\right),\left(\hat{w}, \hat{w}_{2}\right)\right)=\mathcal{B}\left(\left(\nabla \hat{\psi}, \boldsymbol{\psi}_{1}, \hat{\psi}_{2}\right),\left(\nabla \hat{w}, 0, \hat{w}_{2}\right)\right)
$$

for all $\left(\hat{\psi}, \boldsymbol{\psi}_{1}, \hat{\psi}_{2}\right) \in H_{0}^{1}(\Omega) \times L^{2}\left(\Omega ; \mathbf{H}_{\sharp, 0}^{1}(Y)\right) \times L^{2}\left(\Omega ; H_{\sharp, 0}^{1}(Y)\right)$. Inserting $\hat{\psi}=\theta$ and $\hat{\psi}_{2}=e_{2}$, we then obtain

$$
\begin{aligned}
\|\theta\|_{L^{2}}^{2} & =\mathcal{A}\left(\left(\theta, e_{2}\right),\left(\hat{w}, \hat{w}_{2}\right)\right)=\mathcal{B}\left(\left(\nabla \theta, e_{1}, e_{2}\right),\left(\nabla \hat{w}, 0, \hat{w}_{2}\right)\right) \\
& =\mathcal{B}\left(\left(e_{0}, e_{1}, e_{2}\right),\left(\nabla \hat{w}, 0, \hat{w}_{2}\right)\right)-\mathcal{B}\left((\mathbf{z}, 0,0),\left(\nabla \hat{w}, 0, \hat{w}_{2}\right)\right)
\end{aligned}
$$

By the properties of the Helmholtz decomposition we have $\left(\mathbf{z}, \nabla \hat{w}_{H}\right)_{L^{2}}=0$. With

Copyright (c) by SIAM. Unauthorized reproduction of this article is prohibited. 
the same computations as for the dual problem with $\mathbf{z}$, we then derive

$$
\begin{aligned}
\|\theta\|_{L^{2}}^{2}= & \mathcal{B}\left(\left(e_{0}-\mathbf{z}, e_{1}, e_{2}\right),\left(\nabla\left(\hat{w}-\hat{w}_{H}\right), 0, \hat{w}_{2}-\hat{w}_{h}\right)\right)-\mathcal{B}\left((\mathbf{z}, 0,0),\left(\nabla \hat{w}, 0, \hat{w}_{2}\right)\right) \\
& \left.+\left(\mathcal{B}_{h}-\mathcal{B}\right)\left(\mathbf{E}_{H}, \mathbf{K}_{h, 1}\left(\mathbf{E}_{H}\right), K_{h, 2}\left(\mathbf{E}_{H}\right)\right),\left(\nabla \hat{w}_{H}, 0, \hat{w}_{h}\right)\right) \\
\leq & \left|\mathcal{A}\left(\left(\theta, e_{2}\right)\left(\hat{w}-\hat{w}_{H}, \hat{w}_{2}-\hat{w}_{h}\right)\right)\right|+C\|\mathbf{z}\|_{L^{2}}\left\|\nabla \hat{w}+\nabla_{y} \hat{w}_{2}\right\|_{L^{2}(\Omega \times Y)} \\
& +C \max \left\{\left\|\mu^{-1}-\mu_{h}^{-1}\right\|_{L^{\infty}(\Omega \times Y)},\left\|\kappa-\kappa_{h}\right\|_{L^{\infty}(\Omega \times Y)}\right\} \\
& \left\|\left(\mathbf{E}_{H}, \mathbf{K}_{h, 1}\left(\mathbf{E}_{H}\right), K_{h, 2}\left(\mathbf{E}_{H}\right)\right)\right\|_{e}\left\|\nabla \hat{w}_{H}+\nabla_{y} \hat{w}_{h}\right\|_{L^{2}(\Omega \times Y)} \\
\leq & C\left\|\nabla \theta+\nabla_{y} e_{2}\right\|_{L^{2}(\Omega \times Y)}\left\|\nabla\left(\hat{w}-\hat{w}_{H}\right)+\nabla_{y}\left(\hat{w}_{2}-\hat{w}_{h}\right)\right\|_{L^{2}(\Omega \times Y)} \\
& +C \eta_{\text {approx }}\|\theta\|_{L^{2}}\|\mathbf{f}\|_{L^{2}}+C\left\|_{\mathbf{z}}\right\|_{L^{2}}\|\theta\|_{L^{2}},
\end{aligned}
$$

where in the last inequality we used the stability estimate for $\mathbf{E}_{H}$ and a stability estimate for the solution of elliptic two-scale equations. From a priori error estimates for elliptic two-scale problems (see $[27,38]$ ), we know that

$$
\left\|\nabla\left(\hat{w}-\hat{w}_{H}\right)+\nabla_{y}\left(\hat{w}_{2}-\hat{w}_{h}\right)\right\| \leq C(H+h)\|\theta\|_{L^{2}} .
$$

Inserting this, the estimate for the Helmholtz decomposition (6.3), and the estimate for $\mathbf{z}$ from above, we finally obtain

$$
\begin{aligned}
\|\theta\|_{L^{2}}^{2} \leq & C(H+h)\|\theta\|_{L^{2}}\left(\left\|e_{0}\right\|_{L^{2}(\Omega)}+\left\|\nabla_{y} e_{2}\right\|_{L^{2}}\right) \\
& +C(H+h)\left\|\left(e_{0}, e_{1}, e_{2}\right)\right\|_{e}\|\theta\|_{L^{2}}+C \eta_{\text {approx }}\|\theta\|_{L^{2}}\|\mathbf{f}\|_{L^{2}} .
\end{aligned}
$$

The estimates for $\mathbf{z}$ and $\theta$ together with the a priori error estimate of Theorem 5.2 give the claim.

6.2. Proofs of the a posteriori estimates. For the a posteriori estimates we no longer assume higher regularity and therefore need other interpolation operators.

Lemma 6.3 (Clément and Schöberl interpolation operators). Denote by $I_{h}$ : $L^{2}\left(\Omega ; L^{2}(Y)\right) \rightarrow L^{2}\left(\Omega ; \widetilde{W}_{h}^{1}(Y)\right)$ the Clément interpolation operator with appropriate adaptations to periodic boundary conditions and zero average. Then the following estimates hold for all $u \in L^{2}\left(\Omega ; H_{\sharp, 0}^{1}(Y)\right)$ :

$$
\begin{aligned}
\left\|u-I_{h} u\right\|_{L^{2}\left(T_{j} \times S_{k}\right)} & \leq C h_{k}\left\|\nabla_{y} u\right\|_{L^{2}\left(T \times \omega_{S_{k}}\right)}, \\
\left\|u-I_{h} u\right\|_{L^{2}\left(T_{j} \times \tilde{F}_{k m}\right)} & \leq C h_{k m}^{1 / 2}\left\|\nabla_{y} u\right\|_{L^{2}\left(T \times \omega_{\tilde{F}_{k m}}\right)} .
\end{aligned}
$$

Again, the Clément operator can be defined componentwise for vector functions and then fulfills the same estimates. Denote by $I_{H}: \mathbf{H}_{0}(\mathrm{curl}) \rightarrow \mathbf{V}_{H, 0}^{I}$ the Schöberl interpolation operator. For every $\mathbf{u} \in \mathbf{H}_{0}(\mathrm{curl})$ there exist $\theta \in H_{0}^{1}(\Omega)$ and $\mathbf{z} \in \mathbf{H}_{0}^{1}(\Omega)$ with $\mathbf{u}-I_{H} \mathbf{u}=\nabla \theta+\mathbf{z}$ such that the decomposition fulfills the estimates

$$
\begin{aligned}
& H_{j}^{-1}\|\theta\|_{L^{2}\left(T_{j}\right)}+\|\nabla \theta\|_{L^{2}\left(T_{j}\right)} \leq C\|\mathbf{u}\|_{L^{2}\left(\tilde{\omega}_{T_{j}}\right)}, \\
& H_{j}^{-1}\|\mathbf{z}\|_{L^{2}\left(T_{j}\right)}+\|\nabla \mathbf{z}\|_{L^{2}\left(T_{j}\right)} \leq C\|\operatorname{curl} \mathbf{u}\|_{L^{2}\left(\tilde{\omega}_{T_{j}}\right)}
\end{aligned}
$$

and, together with the trace inequality,

$$
\|\theta\|_{L^{2}\left(F_{j l}\right)} \leq C H_{j l}^{1 / 2}\|\mathbf{u}\|_{L^{2}\left(\tilde{\omega}_{T_{j}}\right)}, \quad \text { and } \quad\|\mathbf{z}\|_{L^{2}\left(F_{j l}\right)} \leq C H_{j l}^{1 / 2}\|\operatorname{curl} \mathbf{u}\|_{L^{2}\left(\tilde{\omega}_{T_{j}}\right)} .
$$

Proof. A proof for $I_{h}$ can be found in [15] and for $I_{H}$ in [40]. 
Proof of Theorem 5.4. First, we derive an error identity. From the definition of the error terms and Proposition 4.3 we deduce

$$
\begin{aligned}
& \mathcal{B}\left(\left(e_{0}, e_{1}, e_{2}\right),\left(\boldsymbol{\psi}, \boldsymbol{\psi}_{1}, \psi_{2}\right)\right) \\
&= \mathcal{B}\left(\left(\mathbf{E}, \mathbf{K}_{1}, K_{2}\right),\left(\boldsymbol{\psi}, \boldsymbol{\psi}_{1}, \psi_{2}\right)\right) \\
&-\mathcal{B}\left(\left(\mathbf{E}_{H}, \mathbf{K}_{h, 1}\left(\mathbf{E}_{H}\right), K_{h, 2}\left(\mathbf{E}_{H}\right)\right),\left(\boldsymbol{\psi}, \boldsymbol{\psi}_{1}, \psi_{2}\right)\right) \\
&=(\mathbf{f}, \boldsymbol{\psi})-\mathcal{B}\left(\left(\mathbf{E}_{H}, \mathbf{K}_{h, 1}\left(\mathbf{E}_{H}\right), K_{h, 2}\left(\mathbf{E}_{H}\right)\right),\left(\boldsymbol{\psi}, \boldsymbol{\psi}_{1}, \psi_{2}\right)\right) \\
&-\mathcal{B}_{h}\left(\left(\mathbf{E}_{H}, \mathbf{K}_{h, 1}\left(\mathbf{E}_{H}\right), K_{h, 2}\left(\mathbf{E}_{H}\right)\right),\left(\boldsymbol{\psi}-\boldsymbol{\psi}_{H}, \boldsymbol{\psi}_{1}-\boldsymbol{\psi}_{h}, \psi_{2}-\psi_{h}\right)\right) \\
&+\mathcal{B}_{h}\left(\left(\mathbf{E}_{H}, \mathbf{K}_{h, 1}\left(\mathbf{E}_{H}\right), K_{h, 2}\left(\mathbf{E}_{H}\right)\right),\left(\boldsymbol{\psi}, \boldsymbol{\psi}_{1}, \psi_{2}\right)\right) \\
&-\underbrace{\mathcal{B}_{h}\left(\left(\left(\mathbf{E}_{H}, \mathbf{K}_{h, 1}\left(\mathbf{E}_{H}\right), K_{h, 2}\left(\mathbf{E}_{H}\right)\right),\left(\boldsymbol{\psi}_{H}, \boldsymbol{\psi}_{h}, \psi_{h}\right)\right)\right.}_{=\left(\mathbf{f}, \boldsymbol{\psi}_{H}\right)_{L^{2}}} .
\end{aligned}
$$

With the definition of the residual (6.2), this gives the following error identity for all $\left(\boldsymbol{\psi}, \boldsymbol{\psi}_{1}, \psi_{2}\right) \in \mathbf{H}_{0}(\operatorname{curl}) \times L^{2}\left(\Omega ; \mathbf{H}_{\sharp, 0}^{1}(Y)\right) \times L^{2}\left(\Omega ; H_{\sharp, 0}^{1}(Y)\right)$ and all $\left(\boldsymbol{\psi}_{H}, \boldsymbol{\psi}_{h}, \psi_{h}\right) \in$ $\mathbf{V}_{H, 0}^{I} \times L^{2}\left(\Omega ; \widetilde{\mathbf{W}}_{h}^{1}(Y)\right) \times L^{2}\left(\Omega ; \widetilde{W}_{h}^{1}(Y)\right)$ :

$$
\begin{aligned}
\mathcal{B}\left(\left(e_{0}, e_{1}, e_{2}\right),\left(\boldsymbol{\psi}, \boldsymbol{\psi}_{1}, \psi_{2}\right)\right) & \\
= & -\left\langle\operatorname{Res}_{h}\left(\mathbf{E}_{H}, \mathbf{K}_{h, 1}\left(\mathbf{E}_{H}\right), K_{h, 2}\left(\mathbf{E}_{H}\right)\right),\left(\boldsymbol{\psi}-\boldsymbol{\psi}_{H}, \boldsymbol{\psi}_{1}-\boldsymbol{\psi}_{h}, \psi_{2}-\psi_{h}\right)\right\rangle \\
& +\left(\mathcal{B}_{h}-\mathcal{B}\right)\left(\left(\mathbf{E}_{H}, \mathbf{K}_{h, 1}\left(\mathbf{E}_{H}\right), K_{h, 2}\left(\mathbf{E}_{H}\right)\right),\left(\boldsymbol{\psi}, \boldsymbol{\psi}_{1}, \psi_{2}\right)\right) .
\end{aligned}
$$

We choose $\boldsymbol{\psi}=e_{0}, \boldsymbol{\psi}_{1}=e_{1}, \psi_{2}=e_{2}$ and $\boldsymbol{\psi}_{H}=I_{H} e_{0}, \boldsymbol{\psi}_{h}=I_{h} e_{1}, \psi_{h}=I_{h} e_{2}$ in the error identity (6.4) with the interpolation operators $I_{H}$ and $I_{h}$ from Lemma 6.3. Using the coercivity of $\mathcal{B}$, we obtain

$$
\begin{aligned}
&\left\|\left(e_{0}, e_{1}, e_{2}\right)\right\|_{e}^{2} \\
& \leq C(\underbrace{\left|\left\langle\operatorname{Res}_{h}\left(\mathbf{E}_{H}, \mathbf{K}_{h, 1}\left(\mathbf{E}_{H}\right), K_{h, 2}\left(\mathbf{E}_{H}\right)\right),\left(e_{0}-I_{H} e_{0}, e_{1}-I_{h} e_{1}, e_{2}-I_{h} e_{2}\right)\right\rangle\right|}_{:=\mathrm{I}} \\
&+\underbrace{\left|\left(\mathcal{B}_{h}-\mathcal{B}\right)\left(\left(\mathbf{E}_{H}, \mathbf{K}_{h, 1}\left(\mathbf{E}_{H}\right), K_{h, 2}\left(\mathbf{E}_{H}\right)\right),\left(e_{0}, e_{1}, e_{2}\right)\right)\right|}_{:=\mathrm{II}}) .
\end{aligned}
$$

To estimate I, we insert the decomposition of $e_{0}-I_{H} e_{0}=\nabla \theta+\mathbf{z}$ according to Lemma 6.3 and thus obtain

$$
\begin{aligned}
\mathrm{I}=\mid \int_{\Omega} \int_{Y} \mu_{h}^{-1}\left(\operatorname{curl} \mathbf{E}_{H}+\operatorname{curl}_{y} \mathbf{K}_{h, 1}\right) \cdot\left(\operatorname{curl} \mathbf{z}^{*}+\operatorname{curl}_{y}\left(e_{1}-I_{h} e_{1}\right)^{*}\right) \\
\quad+\operatorname{div}_{y} \mathbf{K}_{h, 1} \operatorname{div}_{y}\left(e_{1}-I_{h} e_{1}\right)^{*} \\
\quad-\kappa_{h}\left(\mathbf{E}_{H}+\nabla_{y} K_{h, 2}\right) \cdot\left(\nabla \theta^{*}+\mathbf{z}^{*}+\nabla_{y}\left(e_{2}-I_{h} e_{2}\right)^{*}\right) d y d x \\
\quad-\int_{\Omega} \mathbf{f}\left(\nabla \theta^{*}+\mathbf{z}^{*}\right) d x \mid .
\end{aligned}
$$

Copyright $@$ by SIAM. Unauthorized reproduction of this article is prohibited. 
Integrating by parts locally, inserting $\mathbf{f}_{H}-\mathbf{f}_{H}$, and noting $\operatorname{div} \mathbf{f}=0$ yields

$$
\begin{aligned}
\mathrm{I}= & \mid \sum_{j \in J} \int_{T_{j}} \sum_{k \in I} \int_{S_{k}} \operatorname{curl}_{x}\left(\mu_{h}^{-1}\left(\operatorname{curl} \mathbf{E}_{H}+\operatorname{curl}_{y} \mathbf{K}_{h, 1}\right)\right) \cdot \mathbf{z}^{*} d y d x \\
& +\sum_{j \in J} \int_{\partial T_{j}} \sum_{k \in I} \int_{S_{k}}\left(\mu_{h}^{-1}\left(\operatorname{curl} \mathbf{E}_{H}+\operatorname{curl}_{y} \mathbf{K}_{h, 1}\right)\right) \times \mathbf{n} \cdot \mathbf{z}^{*} d y d \sigma \\
& +\sum_{j \in J} \int_{T_{j}} \sum_{k \in I} \int_{S_{k}} \operatorname{curl}_{y}\left(\mu_{h}^{-1}\left(\operatorname{curl} \mathbf{E}_{H}+\operatorname{curl}_{y} \mathbf{K}_{h, 1}\right)\right) \cdot\left(e_{1}-I_{h} e_{1}\right)^{*} d y d x \\
& +\sum_{j \in J} \int_{T_{j}} \sum_{k \in I} \int_{\partial S_{k}}\left(\mu_{h}^{-1}\left(\operatorname{curl} \mathbf{E}_{H}+\operatorname{curl}_{y} \mathbf{K}_{h, 1}\right)\right) \times \mathbf{n} \cdot\left(e_{1}-I_{h} e_{1}\right)^{*} d \sigma d x \\
& -\sum_{j \in J} \int_{T_{j}} \sum_{k \in I} \int_{S_{k}} \nabla_{y}\left(\operatorname{div}_{y} \mathbf{K}_{h, 1}\right) \cdot\left(e_{1}-I_{h} e_{1}\right)^{*} d y d x \\
& +\sum_{j \in J} \int_{T_{j}} \sum_{k \in I} \int_{\partial S_{k}} \operatorname{div}_{y} \mathbf{K}_{h, 1} \mathbf{n} \cdot\left(e_{1}-I_{h} e_{1}\right)^{*} d \sigma d x \\
& +\sum_{j \in J} \int_{T_{j}} \sum_{k \in I} \int_{S_{k}} \operatorname{div}_{x}\left(\kappa_{h}\left(\mathbf{E}_{H}+\nabla_{y} K_{h, 2}\right)\right) \theta^{*} d y d x \\
& -\sum_{j \in J} \int_{\partial T_{j}} \sum_{k \in I} \int_{S_{k}}\left(\kappa_{h}\left(\mathbf{E}_{H}+\nabla_{y} K_{h, 2}\right)\right) \cdot \mathbf{n} \theta^{*} d y d \sigma \\
& +\sum_{j \in J} \int_{T_{j}} \sum_{k \in I} \int_{S_{k}} \operatorname{div}_{y}\left(\kappa_{h}\left(\mathbf{E}_{H}+\nabla_{y} K_{h, 2}\right)\right) \cdot\left(e_{2}-I_{h} e_{2}\right)^{*} d y d x \\
& -\sum_{j \in J} \int_{T_{j}} \sum_{k \in I} \int_{\partial S_{k}}\left(\kappa_{h}\left(\mathbf{E}_{H}+\nabla_{y} K_{h, 2}\right)\right) \cdot \mathbf{n}\left(e_{2}-I_{h} e_{2}\right)^{*} d \sigma d x \\
& -\sum_{j \in J} \int_{T_{j}} \sum_{k \in I} \int_{S_{k}}\left(\mathbf{f}_{H}+\mathbf{f}-\mathbf{f}_{H}+\kappa_{h}\left(\mathbf{E}_{H}+\nabla_{y} K_{h, 2}\right)\right) \cdot \mathbf{z}^{*} d y d x \mid
\end{aligned}
$$

As $\mu_{h}^{-1}, \kappa_{h}$ are constant on the cells $T_{j} \times S_{k}$, the correctors $K_{h}$ are linear with respect to $y, \mathbf{K}_{h, 1}$ is constant with respect to $x$, and $\mathbf{E}_{H}$ is linear with respect to $x$ and all terms with two derivatives with respect to the same variable cancel out. We derive by a rearrangement of sums and the Hölder inequality

$$
\begin{aligned}
\mathrm{I} \leq & \sum_{(j, l) \in \mathcal{E}\left(\mathcal{T}_{H}\right)}\left\|\left[\int_{Y}\left(\mu_{h}^{-1}\left(\operatorname{curl} \mathbf{E}_{H}+\operatorname{curl}_{y} \mathbf{K}_{h, 1}\right)\right) \times \mathbf{n} d y\right]_{F_{j l}}\right\|\|\mathbf{z}\|_{L^{2}\left(F_{j l}\right)} \\
& +\sum_{(j, l) \in \mathcal{E}\left(\mathcal{T}_{H}\right)}\left\|\left[\int_{Y}\left(\kappa_{h}\left(\mathbf{E}_{H}+\nabla_{y} K_{h, 2}\right)\right) \cdot \mathbf{n} d y\right]_{F_{j l}}\right\|\|\theta\|_{L^{2}\left(F_{j l}\right)} \\
& +\sum_{j \in J} \sum_{(k, m) \in \mathcal{E}\left(\mathcal{T}_{h}\right)}\left\|\left[\left(\mu_{h}^{-1}\left(\operatorname{curl} \mathbf{E}_{H}+\operatorname{curl}_{y} \mathbf{K}_{h, 1}\right)\right) \times \mathbf{n}+\operatorname{div}_{y} \mathbf{K}_{h, 1} \mathbf{n}\right]_{\tilde{F}_{k m}}\right\| \\
& \left.\left.+\sum_{j \in J} \sum_{(k, m) \in \mathcal{E}\left(\mathcal{T}_{h}\right)} \|\left[\left(\kappa_{1}-I_{h} e_{1}\right) \|_{L^{2}\left(\mathbf{E}_{j} \times \tilde{F}_{k m}\right)}+\nabla_{y} K_{h, 2}\right)\right) \cdot \mathbf{n}\right]_{\tilde{F}_{k m}}\|\|\left(e_{2}-I_{h} e_{2}\right) \|_{L^{2}\left(T_{j} \times \tilde{F}_{k m}\right)}
\end{aligned}
$$

Copyright $@$ by SIAM. Unauthorized reproduction of this article is prohibited. 


$$
\begin{aligned}
& +\sum_{j \in J}\left\|\operatorname{div}_{x}\left(\int_{Y} \kappa_{h}\left(\mathbf{E}_{H}+\nabla_{y} K_{h, 2}\right) d y\right)\right\|\|\theta\|_{L^{2}\left(T_{j}\right)} \\
& +\sum_{j \in J}\left(\left\|\mathbf{f}-\mathbf{f}_{H}\right\|+\left\|\mathbf{f}_{H}+\int_{Y} \kappa_{h}\left(\mathbf{E}_{H}+\nabla_{y} K_{h, 2}\right) d y\right\|\right)\|\mathbf{z}\|_{L^{2}\left(T_{j}\right)} .
\end{aligned}
$$

Using the estimates for the interpolation operators from Lemma 6.3, we get

$$
\begin{aligned}
\mathrm{I} \leq & \sum_{(j, l) \in \mathcal{E}\left(\mathcal{T}_{H}\right)} C H_{j l}^{1 / 2}\left\|\left[\int_{Y}\left(\mu_{h}^{-1}\left(\operatorname{curl} \mathbf{E}_{H}+\operatorname{curl}_{y} \mathbf{K}_{h, 1}\right)\right) \times \mathbf{n} d y\right]_{F_{j l}}\right\| \\
& +\sum_{(j, l) \in \mathcal{E}\left(\mathcal{T}_{H}\right)} C H_{j l}^{1 / 2}\left\|\left[\int_{Y}\left(\kappa_{h}\left(\mathbf{E}_{H}+\nabla_{y} K_{h, 2}\right)\right) \cdot \mathbf{n} d y\right]_{F_{j l} l}\right\|\left\|e_{0}\right\|_{L^{2}\left(\tilde{\omega}_{T_{j}}\right)} \\
& +\sum_{j \in J(k, m) \in \mathcal{E}\left(\tilde{T}_{h}\right)} C h_{k m}^{1 / 2}\left\|\left[\left(\mu_{h}^{-1}\left(\operatorname{curl} \mathbf{E}_{H}+\operatorname{curl}_{y} \mathbf{K}_{h, 1}\right)\right) \times \mathbf{n}+\operatorname{div}_{y} \mathbf{K}_{h, 1} \mathbf{n}\right]_{\tilde{F}_{k m}}\right\| \\
& \left.+\sum_{j \in J} \sum_{(k, m) \in \mathcal{E}\left(\mathcal{T}_{h}\right)} C h_{k m}^{1 / 2}\left\|\left[\left(\kappa_{h}\left(\mathbf{E}_{H}+\nabla_{y} K_{h, 2}\right)\right) \cdot \mathbf{n}\right]_{\tilde{F}_{k m}}\right\|\left\|\nabla_{y} e_{2}\right\|_{L^{2}\left(T_{j} \times \omega_{\tilde{F}_{k m}}\right)}\right) \\
& +\sum_{j \in J} C H_{j}\left\|\operatorname{div}_{x}\left(\int_{Y} \kappa_{h}\left(\mathbf{E}_{H}+\nabla_{y} K_{h, 2}\right) d y\right)\right\|\left\|e_{0}\right\|_{L^{2}\left(\tilde{\omega}_{T_{j}}\right)} \\
& +\sum_{j \in J} C H_{j}\left(\left\|\mathbf{f}-\mathbf{f}_{H}\right\|+\left\|\mathbf{f}_{H}+\int_{Y} \kappa_{h}\left(\mathbf{E}_{H}+\nabla_{y} K_{h, 2}\right) d y\right\|\right) \\
& \left\|\operatorname{curl} e_{0}\right\|_{L^{2}\left(\tilde{\omega}_{T_{j}}\right)} .
\end{aligned}
$$

Applying the Cauchy-Schwarz inequality, we obtain the desired local estimators and terms like $\sum_{j \in J}\left\|\operatorname{curl} e_{0}\right\|_{L^{2}\left(\tilde{\omega}_{T_{j}}\right)}$. As the triangulation is shape regular, each element $T_{j}$ only appears in a finite number of these neighborhoods and this number can be bounded above by a uniform constant (independent of $H, h$ ). Thus, we derive

$$
\begin{aligned}
\mathrm{I} \leq & C\left(\sum_{(j, l) \in \mathcal{E}\left(\mathcal{T}_{H}\right)} \eta_{j l, 1}^{2}\right)^{1 / 2}\left\|\operatorname{curl} e_{0}\right\|_{L^{2}(\Omega)}+C\left(\sum_{(j, l) \in \mathcal{E}\left(\mathcal{T}_{H}\right)} \eta_{j l, 2}^{2}\right)^{1 / 2}\left\|e_{0}\right\|_{L^{2}(\Omega)} \\
& +C\left(\sum_{j \in J} \sum_{(k, m) \in \mathcal{E}\left(\mathcal{T}_{h}\right)} \eta_{j, k m, 1}^{2}\right)^{1 / 2}\left\|\nabla_{y} e_{1}\right\|_{L^{2}(\Omega \times Y)} \\
& +C\left(\sum_{j \in J} \sum_{(k, m) \in \mathcal{E}\left(\mathcal{T}_{h}\right)} \eta_{j, k m, 2}^{2}\right)^{1 / 2}\left\|\nabla_{y} e_{2}\right\|_{L^{2}(\Omega \times Y)} \\
& +C\left(\sum_{j \in J} \eta_{j, 2}^{2}\right)^{1 / 2}\left\|e_{0}\right\|_{L^{2}(\Omega)}+C\left(\sum_{j \in J} \zeta_{j}^{2}+\eta_{j, 1}^{2}\right)^{1 / 2}\left\|\operatorname{curl} e_{0}\right\|_{L^{2}(\Omega)} .
\end{aligned}
$$

All norms of the errors $e_{0}, e_{1}$, and $e_{2}$ can simply be estimated by $\left\|\left(e_{0}, e_{1}, e_{2}\right)\right\|_{e}$.

Copyright $@$ by SIAM. Unauthorized reproduction of this article is prohibited. 
To estimate II, we just split the integral into local terms and use the Hölder inequality

$$
\begin{aligned}
\mathrm{II}= & \mid \sum_{j \in J} \int_{T_{j}} \sum_{k \in I} \int_{S_{k}}\left(\mu^{-1}\left(x_{j}, y_{i}\right)-\mu^{-1}(x, y)\right)\left(\operatorname{curl} \mathbf{E}_{H}+\operatorname{curl}_{y} \mathbf{K}_{h, 1}\right) \\
& \cdot\left(\operatorname{curl} e_{0}^{*}+\operatorname{curl}_{y} e_{1}^{*}\right) \\
& +\left(\kappa\left(x_{j}, y_{k}\right)-\kappa(x, y)\right)\left(\mathbf{E}_{H}+\nabla_{y} K_{h, 2}\right) \cdot\left(e_{0}^{*}+\nabla_{y} e_{2}^{*}\right) d y d x \mid \\
\leq & \left(\sum_{j \in J} \sum_{k \in I} \zeta_{j k}^{2}\right)^{1 / 2}\left\|\left(e_{0}, e_{1}, e_{2}\right)\right\|_{e} .
\end{aligned}
$$

Dividing each estimate by $\left\|\left(e_{0}, e_{1}, e_{2}\right)\right\|_{e}$ and combining both gives us the a posteriori error estimate.

For the proof of the lower bound we need local bubble functions. Let us denote by $\lambda_{T, l}, l=1, \ldots, 4$, the barycentric coordinates of a tetrahedron $T$ and by $\lambda_{F, l}$, $l=1,2,3$, the barycentric coordinates of a face $F$. The local bubble functions on elements and faces are defined as

$$
\psi_{T}:=256 \prod_{l=1}^{4} \lambda_{T, l}, \quad \psi_{F}:=27 \prod_{l=1}^{3} \lambda_{F, l} .
$$

They fulfill $0 \leq \psi_{T}, \psi_{F} \leq 1, \operatorname{supp} \psi_{T} \subset T$, and $\operatorname{supp} \psi_{F} \subset \hat{\omega}_{F}$. We also define a continuation operator $P_{F}: L^{\infty}(F) \rightarrow L^{\infty}\left(\hat{\omega}_{F}\right)$ as the constant extension of a function in the direction perpendicular to the face $F$; for details see [43]. The following inequalities can be proved with standard scaling arguments and the properties of the bubble functions. (See [43, Proposition 3.37] for details and the proof.)

Lemma 6.4 (inverse inequalities). For all $g \in \mathbb{P}^{k}$ and all tetrahedra $T$ it holds that

$$
\begin{aligned}
\|g\|_{L^{2}(T)}^{2} & \leq C\left|\left(g, \psi_{T} g\right)_{T}\right| \\
\left\|\psi_{T} g\right\|_{L^{2}(T)} & \leq C\|g\|_{L^{2}(T)}, \\
\left\|\nabla\left(\psi_{T} g\right)\right\|_{L^{2}(T)} & \leq C \operatorname{diam}(T)^{-1}\|g\|_{L^{2}(T)} .
\end{aligned}
$$

Furthermore, for all $\left.f \in \mathbb{P}^{k}\right|_{F}$ and faces $F$ it holds that

$$
\begin{aligned}
\|f\|_{L^{2}(F)}^{2} & \leq C\left|\left(g, \psi_{F} P_{F}(f)\right)_{F}\right| \\
\left\|\psi_{F} P_{F}(f)\right\|_{L^{2}\left(\omega_{F}\right)} & \leq C \operatorname{diam}(F)^{1 / 2}\|f\|_{L^{2}(F)}, \\
\left\|\nabla\left(\psi_{F} P_{F}(f)\right)\right\|_{L^{2}\left(\omega_{F}\right)} & \leq C \operatorname{diam}(F)^{-1 / 2}\|f\|_{L^{2}(F)} .
\end{aligned}
$$

Proof of Theorem 5.5. First, corresponding to the error terms we introduce the following functions:

$$
\begin{aligned}
\mathbf{w}_{j, 1}(x)= & \psi_{T_{j}}(x)\left(\mathbf{f}_{H}+\int_{Y} \kappa_{h}(\cdot, y)\left(\mathbf{E}_{H}+\nabla_{y} K_{h, 2}(\cdot, y)\right) d y\right)(x), \\
\mathbf{w}_{j l, 1}(x)= & \psi_{F_{j l}}(x) P_{F_{j l}}\left(\left[\int_{Y}\left(\mu_{h}^{-1}\left(\operatorname{curl} \mathbf{E}_{H}+\operatorname{curl}_{y} \mathbf{K}_{h, 1}\right)\right) \times \mathbf{n} d y\right]_{F_{j l}}\right)(x), \\
\mathbf{w}_{j, k m, 1}(x, y)= & \chi_{T_{j}}(x) \psi_{\tilde{F}_{k m}}(y) \\
& P_{\tilde{F}_{k m}}\left(\left[\left(\mu_{h}^{-1}\left(\operatorname{curl} \mathbf{E}_{H}+\operatorname{curl}_{y} \mathbf{K}_{h, 1}\right)\right) \times \mathbf{n}+\operatorname{div}_{y} \mathbf{K}_{h, 1} \mathbf{n}\right]_{\tilde{F}_{k m}}\right)(y),
\end{aligned}
$$

Copyright (c) by SIAM. Unauthorized reproduction of this article is prohibited. 
where $\chi_{A}$ denotes the characteristic function of the set $A$. The localized functions $w_{j, 2}, w_{j l, 2}$, and $w_{j, i k, 2}$ are defined in a similar fashion.

Now the error indicators can be estimated separately: By partial integration they can be interpreted as the residual tested with the localized functions $w$ or $\mathbf{w}$, respectively. The error identity and Lemma 6.4 are then used each time to bound the indicators by the total error. We show this for the terms $\eta_{j, 1}, \eta_{j l, 1}$, and $\eta_{j, i k, 1}$ and leave out the proof for the other estimators as the main ideas are similar.

As $\mathbf{E}_{H}$ is linear and $\mathbf{K}_{h, 1}$ constant with respect to $x$, we get with integration by parts and $\operatorname{suppw}_{j, 1} \subset T_{j}$

$$
\begin{aligned}
0 & =\int_{\Omega} \int_{Y} \operatorname{curl}_{x}\left(\mu_{h}^{-1}\left(\operatorname{curl} \mathbf{E}_{H}+\operatorname{curl}_{y} \mathbf{K}_{h, 1}\right)\right) \cdot \mathbf{w}_{j, 1}^{*} d y d x \\
& =\int_{\Omega} \int_{Y} \mu_{h}^{-1}\left(\operatorname{curl} \mathbf{E}_{H}+\operatorname{curl}_{y} \mathbf{K}_{h, 1}\right) \cdot \operatorname{curl}_{j, 1}^{*} d y d x .
\end{aligned}
$$

Therefore, we obtain for the first error indicator

$$
\begin{aligned}
\frac{\eta_{j, 1}^{2}}{H_{j}^{2}} & \leq C\left|\int_{T_{j}}\left(\mathbf{f}_{H}+\int_{Y} \kappa_{h}\left(\mathbf{E}_{H}+\nabla_{y} K_{h, 2}\right) d y\right) \cdot \mathbf{w}_{j, 1}^{*} d x\right| \\
& =C\left|-\left\langle\operatorname{Res}_{h}\left(\mathbf{E}_{H}, \mathbf{K}_{h, 1}, K_{h, 2}\right),\left(\mathbf{w}_{j, 1}, 0,0\right)\right\rangle+\left(\mathbf{f}_{H}-\mathbf{f}, \mathbf{w}_{j, 1}\right)_{L^{2}}\right| .
\end{aligned}
$$

If we choose $\boldsymbol{\psi}=\mathbf{w}_{j, 1}, \psi_{1}=0, \psi_{2}=0$ in the error identity (6.4), we get with Lemma 6.4

$$
\begin{aligned}
\mid- & \left\langle\operatorname{Res}_{h}\left(\mathbf{E}_{H}, \mathbf{K}_{h, 1}, K_{h, 2}\right),\left(\mathbf{w}_{j, 1}, 0,0\right)\right\rangle+\left(\mathbf{f}_{H}-\mathbf{f}, \mathbf{w}_{j, 1}\right)_{L^{2}} \mid \\
= & \mid \mathcal{B}\left(\left(e_{0}, e_{1}, e_{2}\right),\left(\mathbf{w}_{j, 1}, 0,0\right)\right)+\left(\mathcal{B}-\mathcal{B}_{h}\right)\left(\left(\mathbf{E}_{H}, \mathbf{K}_{h, 1}, K_{h, 2}\right),\left(\mathbf{w}_{j, 1}, 0,0\right)\right) \\
& +\left(\mathbf{f}_{H}-\mathbf{f}, \mathbf{w}_{j, 1}\right)_{L^{2}} \mid \\
\leq & C\left\|\left(e_{0}, e_{1}, e_{2}\right)\right\|_{e\left(T_{j} \times Y\right)}\left\|\mathbf{w}_{j, 1}\right\|_{\mathbf{H}\left(\operatorname{curl}, T_{j}\right)}+C\left\|\mathbf{f}_{H}-\mathbf{f}\right\|_{L^{2}\left(T_{j}\right)}\left\|\mathbf{w}_{j, 1}\right\|_{L^{2}\left(T_{j}\right)} \\
& +C\left(\sum_{i \in I} \zeta_{j i}^{2}\right)^{1 / 2}\left\|\mathbf{w}_{j, 1}\right\|_{\mathbf{H}\left(\operatorname{curl}, T_{j}\right)} \\
\leq & C\left\|\mathbf{f}_{H}-\mathbf{f}\right\|_{L^{2}\left(T_{j}\right)} H_{j}^{-1} \eta_{j, 1}+C H_{j}^{-2}\left\|\left(e_{0}, e_{1}, e_{2}\right)\right\|_{e\left(T_{j} \times Y\right)} \eta_{j, 1} \\
& +C H_{j}^{-2}\left(\sum_{i \in I} \zeta_{j i}^{2}\right)^{1 / 2} \eta_{j, 1} .
\end{aligned}
$$

All in all, after multiplying by $H_{j}^{2} \eta_{j, 1}^{-1}$, this gives the local estimate for $\eta_{j, 1}$.

For $\eta_{j l, 1}$ we have the estimate

$$
\frac{\eta_{j l, 1}^{2}}{H_{j l}} \leq C\left|\int_{F_{j l}}\left[\int_{Y}\left(\mu_{h}^{-1}\left(\operatorname{curl} \mathbf{E}_{H}+\operatorname{curl}_{y} \mathbf{K}_{h, 1}\right)\right) \times \mathbf{n} d y\right]_{F_{j l}} \cdot \mathbf{w}_{j l, 1}^{*} d \sigma\right| .
$$

Copyright (c) by SIAM. Unauthorized reproduction of this article is prohibited. 
An integration by parts and the linearity of $\mathbf{E}_{H}$ yield

$$
\begin{aligned}
\int_{F_{j l}} & {\left[\int_{Y}\left(\mu_{h}^{-1}\left(\operatorname{curl} \mathbf{E}_{H}+\operatorname{curl}_{y} \mathbf{K}_{h, 1}\right)\right) \times \mathbf{n} d y\right]_{F_{j l}} \cdot \mathbf{w}_{j l, 1}^{*} d \sigma } \\
= & \int_{\hat{\omega}_{F_{j l}}} \int_{Y} \mu_{h}^{-1}\left(\operatorname{curl} \mathbf{E}_{H}+\operatorname{curl}_{y} \mathbf{K}_{h, 1}\right) \cdot \operatorname{curl} \mathbf{w}_{j l, 1}^{*} d y d x \\
= & \left\langle\operatorname{Res}_{h}\left(\mathbf{E}_{H}, \mathbf{K}_{h, 1}, K_{h, 2}\right),\left(\mathbf{w}_{j l, 1}, 0,0\right)\right\rangle+\int_{\hat{\omega}_{F_{j l}}}\left(\mathbf{f}-\mathbf{f}_{H}\right) \cdot \mathbf{w}_{j l, 1}^{*} d x \\
& +\int_{\hat{\omega}_{F_{j l}}}\left(\mathbf{f}_{H}+\int_{Y} \kappa_{h}\left(\mathbf{E}_{H}+\nabla_{y} K_{h, 2}\right) d y\right) \cdot \mathbf{w}_{j l, 1}^{*} d x .
\end{aligned}
$$

The integrals over $\hat{\omega}_{F_{j l}}$ can be split into two element integrals over $T_{j}$ and $T_{l}$. In the third term we recognize the known error indicator $\eta_{j, 1}$ and in the second term the data approximation error indicator $\zeta_{j}$. Using first the error identity (6.4) with $\boldsymbol{\psi}=\mathbf{w}_{j l, 1}, \boldsymbol{\psi}_{1}=0$, and $\psi_{2}=0$ and Lemma 6.4 , we obtain

$$
\begin{aligned}
\frac{\eta_{j l, 1}^{2}}{H_{j l}} \leq C & \left(\left\|\left(e_{0}, e_{1}, e_{2}\right)\right\|_{e\left(\hat{\omega}_{F_{j l}} \times Y\right)}\left\|\mathbf{w}_{j l, 1}\right\|_{\mathbf{H}\left(\mathrm{curl}, \hat{\omega}_{F_{j l}}\right)}\right. \\
& +\left(\sum_{i} \zeta_{j i}^{2}+\zeta_{l i}^{2}\right)^{1 / 2}\left\|\mathbf{w}_{j l, 1}\right\|_{\mathbf{H}\left(\mathrm{curl}, \hat{\omega}_{F_{j l}}\right)} \\
& +H_{j}^{-1} \eta_{j, 1}\left\|\mathbf{w}_{j l, 1}\right\|_{L^{2}\left(T_{j}\right)}+H_{l}^{-1} \eta_{l, 1}\left\|\mathbf{w}_{j l, 1}\right\|_{L^{2}\left(T_{l}\right)} \\
& \left.+\left\|\mathbf{f}-\mathbf{f}_{H}\right\|_{L^{2}\left(T_{j}\right)}\left\|\mathbf{w}_{j l, 1}\right\|_{L^{2}\left(T_{j}\right)}+\left\|\mathbf{f}-\mathbf{f}_{H}\right\|_{L^{2}\left(T_{l}\right)}\left\|\mathbf{w}_{j l, 1}\right\|_{L^{2}\left(T_{l}\right)}\right) \\
\leq C & \left(\left\|\left(e_{0}, e_{1}, e_{2}\right)\right\|_{e\left(\hat{\omega}_{F_{j l}} \times Y\right)} H_{j l}^{-1} \eta_{j l, 1}+H_{j l}^{-1}\left(\sum_{i} \zeta_{j i}^{2}+\zeta_{l i}^{2}\right)^{1 / 2} \eta_{j l, 1}\right. \\
& \left.+\left(H_{j}^{-1} \eta_{j, 1}+H_{l}^{-1} \eta_{l, 1}\right) \eta_{j l, 1}+\left(H_{j}^{-1} \zeta_{j}+H_{l}^{-1} \zeta_{l}\right) \eta_{j l, 1}\right)
\end{aligned}
$$

Due to the regularity of the triangulation the quotients $H_{j l} / H_{j}$ and $H_{j l} / H_{l}$ can be bounded above and below. Thus, multiplication by $H_{j l} \eta_{j l, 1}^{-1}$ together with the already derived estimate for $\eta_{j, 1}$ yields the desired estimate for $\eta_{j l, 1}$.

For $\eta_{j, k m, 1}$ we have the estimate

$$
\begin{aligned}
& \frac{\eta_{j, k m, 1}^{2}}{h_{k m}} \leq C \mid \int_{T_{j}} \int_{\tilde{F}_{k m}}\left[\left(\mu_{h}^{-1}\left(\operatorname{curl} \mathbf{E}_{H}+\operatorname{curl}_{y} \mathbf{K}_{h, 1}\right)\right) \times \mathbf{n}+\operatorname{div}_{y} \mathbf{K}_{h, 1} \mathbf{n}\right]_{\tilde{F}_{k m}} \\
& \cdot \mathbf{w}_{j, i k, 1}^{*} d \sigma d x \mid \cdot
\end{aligned}
$$

With an integration by parts and the linearity of $\mathbf{K}_{h, 1}$ with respect to $y$ we obtain

$$
\begin{aligned}
& \int_{T_{j}} \int_{\tilde{F}_{k m}}\left[\left(\mu_{h}^{-1}\left(\operatorname{curl} \mathbf{E}_{H}+\operatorname{curl}_{y} \mathbf{K}_{h, 1}\right)\right) \times \mathbf{n}+\operatorname{div}_{y} \mathbf{K}_{h, 1} \mathbf{n}\right]_{\tilde{F}_{k m}} \cdot \mathbf{w}_{j, i k, 1}^{*} d \sigma d x \\
& =\int_{T_{j}} \int_{\hat{\omega}_{\tilde{F}_{k m}}} \mu_{h}^{-1}\left(\operatorname{curl} \mathbf{E}_{H}+\operatorname{curl}_{y} \mathbf{K}_{h, 1}\right) \cdot \operatorname{curl}_{y} \mathbf{w}_{j, k m, 1}^{*}+\operatorname{div}_{y} \mathbf{K}_{h, 1} \operatorname{div}_{y} \mathbf{w}_{j, k m, 1}^{*} d y d x \\
& =\left\langle\operatorname{Res}_{h}\left(\mathbf{E}_{H}, \mathbf{K}_{h, 1}, K_{h, 2}\right),\left(0, \mathbf{w}_{j, k m, 1}, 0\right)\right\rangle .
\end{aligned}
$$

Copyright (c) by SIAM. Unauthorized reproduction of this article is prohibited. 
Inserting the error identity (6.4) with $\boldsymbol{\psi}=0=\psi_{2}, \boldsymbol{\psi}_{1}=\mathbf{w}_{j, k m, 1}$ and using Lemma 6.4, we obtain

$$
\begin{aligned}
\frac{\eta_{j, k m, 1}^{2} \leq}{h_{k m}} \leq & C\left(\left\|\left(e_{0}, e_{1}, e_{2}\right)\right\|_{e\left(T_{j} \times \hat{\omega}_{\tilde{F}_{k m}}\right.}\left\|\nabla \mathbf{w}_{j, k m, 1}\right\|_{L^{2}\left(T_{j} \times \omega_{\tilde{F}_{k m}}\right)}\right. \\
& \left.+\left(\zeta_{j k}^{2}+\zeta_{j m}^{2}\right)^{1 / 2}\left\|\nabla \mathbf{w}_{j, k m, 1}\right\|_{L^{2}\left(T_{j} \times \hat{\omega}_{\tilde{F}_{k m}}\right)}\right) \\
\leq & C\left(h_{k m}^{-1}\left(\zeta_{j k}^{2}+\zeta_{j m}^{2}\right)^{1 / 2} \eta_{j, k m, 1}+h_{k m}^{-1}\left\|\left(e_{0}, e_{1}, e_{2}\right)\right\|_{e\left(T_{j} \times \hat{\omega}_{\tilde{F}_{k m}}\right)} \eta_{j, k m, 1}\right) .
\end{aligned}
$$

The global estimate now follows by summing up the local estimates.

7. Numerical experiment. In this section we analyze the HMM numerically and verify the theoretical a priori estimates given in Theorems 5.2 and 5.3. The model problem is chosen in such a way that the cell problems and the homogenized macroscopic equation can be solved analytically. In the first part of this section, the analytical two-scale solution is used as a reference solution for the error computation. In the second part, we compute a reference solution as direct discretization to the heterogeneous problem (2.2) on a well-resolved mesh. The implementation was done with the module dune-gdt [36] of the DUNE software framework [8, 9].

The parameters $\mu^{-1}$ and $\kappa$ are periodic and quasi-one-dimensional, given by

$$
\mu^{-1}(y)=\frac{1}{2+\cos \left(2 \pi y_{1}\right)} \quad \text { and } \quad \kappa(y)=\left(2+\cos \left(2 \pi y_{1}\right)+i\left(2+\sin \left(2 \pi y_{1}\right)\right)\right)^{-1} .
$$

Cell problem (3.2) then has the solutions

$$
\mathbf{v}_{1}=0, \quad \mathbf{v}_{2}=-\frac{1}{4 \pi} \sin \left(2 \pi y_{1}\right) \mathbf{e}_{3}, \quad \mathbf{v}_{3}=\frac{1}{4 \pi} \sin \left(2 \pi y_{1}\right) \mathbf{e}_{2},
$$

and cell problem (3.3) has the solutions

$$
v_{1}=\frac{1-i}{8 \pi}\left(\sin \left(2 \pi y_{1}\right)-i \cos \left(2 \pi y_{1}\right)\right), \quad v_{2}=v_{3}=0 .
$$

We obtain the homogenized matrices

$$
\left(\mu^{-1}\right)^{\text {hom }}=\operatorname{diag}\left(\frac{1}{\sqrt{3}}, \frac{1}{2}, \frac{1}{2}\right) \quad \text { and } \quad \kappa^{\text {hom }}=\frac{1-i}{4} \mathrm{Id} .
$$

For the computational (macroscopic) domain $\Omega=(0,1)^{3}$ and an appropriate volume term $\mathbf{f}$ the homogenized solution $\mathbf{E}$ is given by

$$
\mathbf{E}(x)=\left(\sin \left(\pi x_{2}\right) \sin \left(\pi x_{3}\right), \sin \left(\pi x_{1}\right) \sin \left(\pi x_{3}\right), \sin \left(\pi x_{1}\right) \sin \left(\pi x_{2}\right)\right)^{T} .
$$

In fact the corresponding $\mathbf{f}$ is similar to $\mathbf{E}$ up to a prefactor, related to the homogenized matrices, in each component. Note that $\mathbf{E}$ has zero tangential traces as required and that $\mu^{-1}, \kappa$, and $\mathbf{f}$ fulfill Assumption 2.1. The macroscopic domain $\Omega$ is triangulated with mesh size $H$, and the unit cube is triangulated with mesh width $h=2 H$. Besides the (absolute) errors we also give the experimental order of convergence (EOC), which is defined for two mesh sizes $H_{1}>H_{2}$ and the corresponding error values $e_{H_{1}}$ and $e_{H_{2}}$ as $\mathrm{EOC}(e):=\ln \left(\frac{e_{H_{1}}}{e_{H_{2}}}\right) / \ln \left(\frac{H_{1}}{H_{2}}\right)$. In the tables, we list the EOC for $H_{1}>H_{2}$ in the row of the smaller mesh size $\mathrm{H}_{2}$.

The energy norm for a two-scale triple $\left(\mathbf{u}, \mathbf{u}_{1}, u_{2}\right)$ in principle consists of the $\mathbf{H}$ (curl)-norm of $\mathbf{u}$ and the $H^{1}(Y)$-semi norms of $\mathbf{u}_{1}$ and $u_{2}$. Table 1 shows the 
TABLE 1

Convergence history and EOC for the energy error between the HMM-approximation and the analytical two-scale solution.

\begin{tabular}{ccccccc}
\hline$H$ & $\left\|e_{0}\right\|_{\mathbf{H}(\text { curl })}$ & $\left\|\nabla_{y} e_{1}\right\|_{\Omega \times Y}$ & $\left\|\nabla_{y} e_{2}\right\|_{\Omega \times Y}$ & $\operatorname{EOC}\left(e_{0}\right)$ & $\operatorname{EOC}\left(e_{1}\right)$ & $\operatorname{EOC}\left(e_{2}\right)$ \\
\hline $1 / 4$ & 0.853277 & 1.17791 & 0.145306 & - & - & - \\
$1 / 6$ & 0.553882 & 0.653899 & 0.107064 & 1.066 & 1.452 & 0.753 \\
$1 / 8$ & 0.412427 & 0.510481 & 0.0826471 & 1.025 & 0.861 & 0.900 \\
$1 / 12$ & 0.273752 & 0.346569 & 0.0563294 & 1.011 & 0.955 & 0.945 \\
\hline \multicolumn{7}{c}{ TABLE 2} \\
Convergence history and EOC for the $L^{2}$ and $H^{-1}(\Omega)$ norm of the macroscopic error.
\end{tabular}

\begin{tabular}{ccccc}
\hline$H$ & $\left\|e_{0}\right\|_{L^{2}(\Omega)}$ & $\left\|\theta_{0.5 H}\right\|_{L^{2}(\Omega)}$ & $\operatorname{EOC}\left(\left\|e_{0}\right\|_{L^{2}}\right)$ & $\operatorname{EOC}\left(\left\|\theta_{0.5 H}\right\|\right)$ \\
\hline $1 / 4$ & 0.289838 & 0.0101565 & - & - \\
$1 / 6$ & 0.198128 & 0.00547722 & 0.938 & 1.523 \\
$1 / 8$ & 0.150247 & 0.00286347 & 0.962 & 2.254 \\
$1 / 12$ & 0.100897 & 0.0013916 & 0.982 & 1.780 \\
\hline
\end{tabular}

behavior of these contributions for the error $\left(e_{0}, e_{1}, e_{2}\right)$ between the analytical twoscale solution $\left(\mathbf{E}, \mathbf{K}_{1}, K_{2}\right)$ and the HMM-approximation $\left(\mathbf{E}_{H}, \mathbf{K}_{h, 1}, K_{h, 2}\right)$ (as defined in section 5) when decreasing $H$ and $h$ simultaneously. One can clearly see a linear decay (the EOC is close to 1 ) for all three parts of the energy error as predicted by Theorem 5.2. In order to verify Theorem 5.3, the Helmholtz decomposition of the error $\mathbf{E}-\mathbf{E}_{H}$ has to be computed. As is well known, the gradient part $\theta \in H_{0}^{1}(\Omega)$ can be characterized as a solution of $(\nabla \theta, \nabla v)=\left(\mathbf{E}-\mathbf{E}_{H}, \nabla v\right)$ for all $v \in H_{0}^{1}(\Omega)$. We solve the variational problem using Lagrange finite elements on a refined macroscopic grid with mesh size $0.5 \mathrm{H}$. The obtained approximation $\theta_{0.5 \mathrm{H}}$ of $\theta$ is considered in Table 2, and we verify the predicted quadratic convergence of Theorem 5.3 (the EOC is close to 2). We emphasize that this consideration of the $H^{-1}(\Omega)$-norm is necessary to obtain a higher convergence order by dual problems: Table 2 reveals that the $L^{2}$ norm only shows linear convergence (the EOC is close to 1). The theoretical reasons for this difference have been discussed in section 5 .

Furthermore, we justify our assumption that the homogenization error can be neglected and show that the correctors are needed to approximate the heterogeneous solution $\mathbf{E}_{\delta}$. For the rather large parameter $\delta=0.2$, we compute an approximate solution $\mathbf{E}_{\delta}$ by a standard discretization with edge elements on a well resolved grid with 82,944 entities. Table 3 shows that the correctors are important parts of the approximation, as predicted in Conclusion 4.4. While the $L^{2}(\Omega)$-norm and $\mathbf{H}(\mathrm{curl})$ semi norm between the reference solution and the macroscopic HMM-approximation $\mathbf{E}_{H}$ almost stagnates (Table 3(a), columns 2-3), the error to the zeroth order approximations $\mathbf{E}_{\mathrm{HMM}}^{0}:=\mathbf{E}_{H}+\nabla_{y} K_{h, 2}(\cdot, \dot{\bar{\delta}})$ and $\operatorname{curl} \mathbf{E}_{\mathrm{HMM}}^{0}:=\operatorname{curl} \mathbf{E}_{H}+\operatorname{curl}_{y} \mathbf{K}_{h, 1}(\cdot, \dot{\bar{\delta}})$ converges (almost) linearly as predicted; see the EOCs in Table 3(b). The convergence slows down slightly in the end because the regime where the modeling error dominates over the discretization error is approached. Those results complement and continue the analysis of [30], where a different test case without an analytically known solution has been studied.

Finally, we visualize the differences between a homogenized and heterogeneous solution and their approximations by the HMM. Figure 1 shows the magnitude of four different fields in the plane $z=0.5$ : The expected homogenized solution $\mathbf{E}$ (top left) on a grid with $H=1 / 12$, the macroscopic part of the HMM-approximation $\mathbf{E}_{H}$ 
TABLE 3

Error between a well-resolved reference solution for $\delta=0.2$ and different parts of the HMMapproximation with the abbreviations $\mathbf{E}_{H M M}^{0}:=\mathbf{E}_{H}+\nabla_{y} K_{h, 2}(\cdot, \dot{\bar{\delta}})$ and $\operatorname{curl} \mathbf{E}_{H M M}^{0}:=\operatorname{curl} \mathbf{E}_{H}+$ $\operatorname{curl}_{y} \mathbf{K}_{h, 1}(\cdot, \dot{\delta})$.

(a) Behavior of the $L^{2}$ norms

\begin{tabular}{ccccc}
\hline$H$ & $\left\|\mathbf{E}_{\delta}-\mathbf{E}_{H}\right\|$ & $\left\|\operatorname{curl}\left(\mathbf{E}_{\delta}-\mathbf{E}_{H}\right)\right\|$ & $\left\|\mathbf{E}_{\delta}-\mathbf{E}_{\mathrm{HMM}}^{0}\right\|$ & $\left\|\operatorname{curl} \mathbf{E}_{\delta}-\operatorname{curl} \mathbf{E}_{\mathrm{HMM}}^{0}\right\|$ \\
\hline $1 / 4$ & 0.926464 & 4.26083 & 0.327821 & 1.50675 \\
$1 / 6$ & 0.896573 & 4.19393 & 0.223441 & 0.96815 \\
$1 / 8$ & 0.890253 & 4.16397 & 0.174372 & 0.734601 \\
$1 / 12$ & 0.883976 & 4.14735 & 0.127625 & 0.486469 \\
\hline
\end{tabular}

(b) Experimental order of convergence

\begin{tabular}{ccc}
\hline$H$ & $\mathrm{EOC}\left(\left\|\mathbf{E}_{\delta}-\mathbf{E}_{\mathrm{HMM}}^{0}\right\|\right)$ & $\mathrm{EOC}\left(\left\|\operatorname{curl} \mathbf{E}_{\delta}-\operatorname{curl} \mathbf{E}_{\mathrm{HMM}}^{0}\right\|\right)$ \\
\hline $1 / 4$ & - & - \\
$1 / 6$ & 0.945 & 1.091 \\
$1 / 8$ & 0.862 & 0.960 \\
$1 / 12$ & 0.770 & 1.016 \\
\hline
\end{tabular}
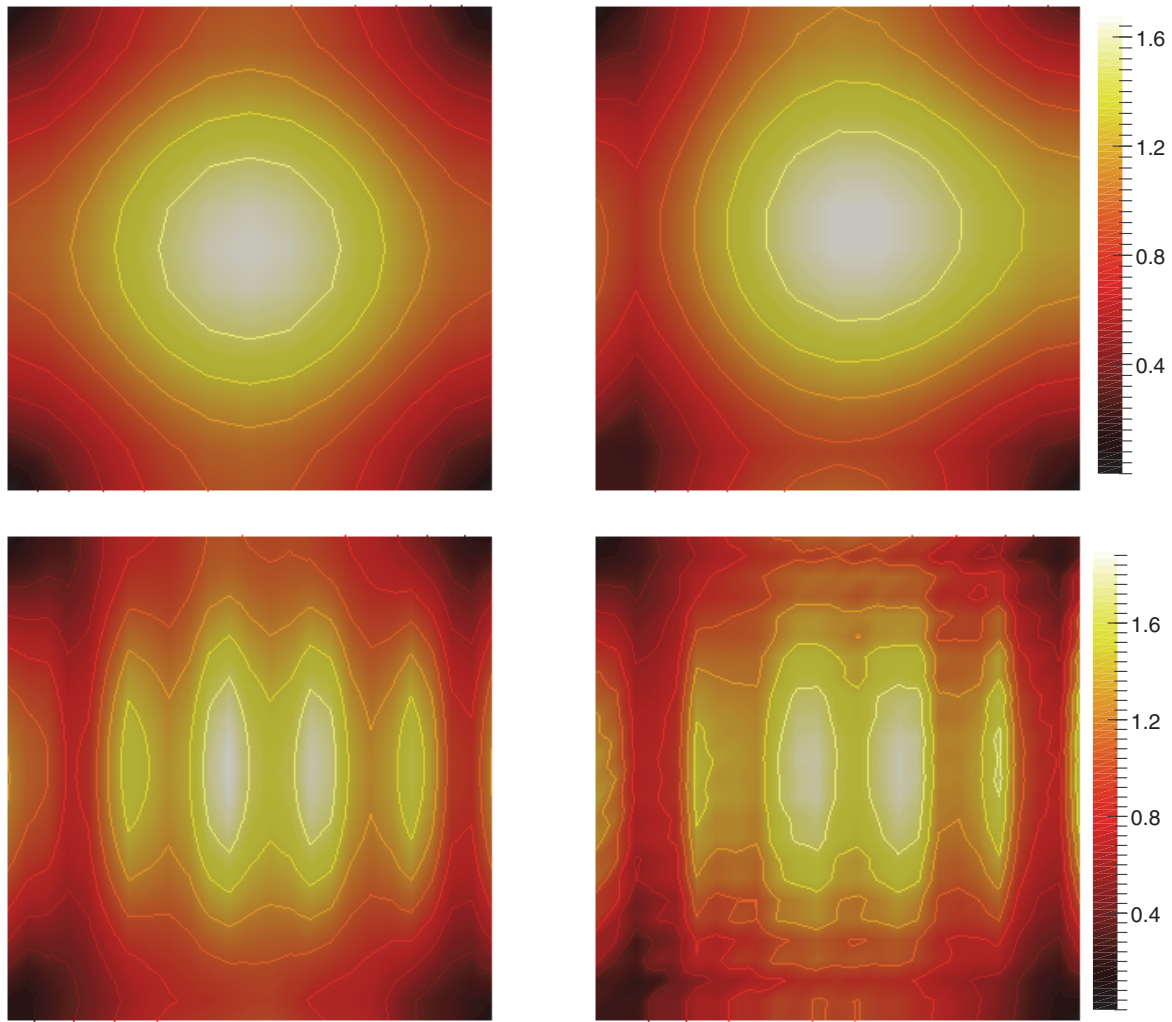

FIG. 1. In the plane $z=0.5$ : Magnitude of the homogenized solution $\mathbf{E}$ (top left), macroscopic part of the HMM-approximation $\mathbf{E}_{H}$ (top right), reference solution on fine grid for $\delta=0.2$ (bottom left), and zeroth order approximation $\mathbf{E}_{H M M}^{0}$ (bottom right).

(top right) for $H=1 / 12$ and $h=1 / 6$, the reference solution $\mathbf{E}_{\delta}$ for $\delta=0.2$ on a mesh with size $H=1 / 24$ (bottom left), and the zeroth order approximation $\mathbf{E}_{\text {нмм }}^{0}$, as defined above, computed with $H=1 / 12$ and $h=1 / 6$ and depicted on the finescale 
reference mesh (bottom right). The figure shows a good correspondence between the HMM-approximation and the expected homogenized or reference solution (the left vs. right picture in the top and bottom row of Figure 1, respectively). Moreover, by comparing the reference solution and the expected homogenized solution (the left column of Figure 1), one can see how the periodic features related to the oscillations in the parameters are in some sense "averaged" in the homogenization procedure.

All in all, the presented numerical results clearly underline the potential of the suggested HMM. The study of even more complex problems such as truly locally periodic problems and the justification of the behavior of the a posteriori error estimators are subjects for future work.

Conclusion. In this paper, we suggested a new HMM for the time-harmonic Maxwell equations. The basis is a homogenization result for a curl-curl-problem obtained with two-scale convergence. Divergence-regularization is applied to the corrector for the curl, and thus we can get rid of the divergence-free constraint. For locally periodic problems, the HMM can be seen as a direct finite element discretization with numerical quadrature of the two-scale homogenized equation, which is the crucial observation for the numerical analysis. The a priori analysis shows that the HMM converges to the homogenized solution with linear or quadratic rate in the energy norm or the $H^{-1}(\Omega)$ norm, respectively, if the meshes are refined simultaneously and the other parameters are fixed (Theorems 5.2 and 5.3). These are the same rates as expected for standard finite elements and time-harmonic Maxwell's equations, but without any preasymptotic effects or conditions on the relation of the mesh width and the periodicity parameter $\delta$. The a posteriori error estimators are reliable and efficient (Theorems 5.4, 5.5) and can be used for adaptive algorithms in future work.

Acknowledgment. We thank the anonymous referees for their valuable remarks which helped to improve the paper.

\section{REFERENCES}

[1] A. AbDulLe, On a priori error analysis of fully discrete heterogeneous multiscale FEM, Multiscale Model. Simul., 4 (2005), pp. 447-459.

[2] A. Abdulle and M. J. Grote, Finite element heterogeneous multiscale method for the wave equation, Multiscale Model. Simul., 9 (2011), pp. 766-792.

[3] A. Abdulle, M. J. Grote, and C. Stohrer, Finite element heterogeneous multiscale method for the wave equation: Long-time effects, Multiscale Model. Simul., 12 (2014), pp. 12301257.

[4] G. Allaire, Homogenization and two-scale convergence, SIAM J. Math. Anal., 23 (1992), pp. $1482-1518$.

[5] Y. Amirat and V. Shelukhin, Homogenization of time harmonic Maxwell equations and the frequency dispersion effect, J. Math. Pures Appl. (9), 95 (2011), pp. 420-443.

[6] D. Arjmand and O. Runborg, Analysis of heterogeneous multiscale methods for long time wave propagation problems, Multiscale Model. Simul., 12 (2014), pp. 1135-1166.

[7] I. BABUŠKA, Error-bounds for finite element method, Numer. Math., 16 (1970/1971), pp. 322333.

[8] P. Bastian, M. Blatt, A. Dedner, C. Engwer, R. Klöfkorn, R. Kornhuber, M. Ohlberger, And O. SAnder, A generic grid interface for parallel and adaptive scientific computing. II. Implementation and tests in DUNE, Computing, 82 (2008), pp. 121138.

[9] P. Bastian, M. Blatt, A. Dedner, C. Engwer, R. Klöfkorn, M. Ohlberger, and O. SANDER, A generic grid interface for parallel and adaptive scientific computing. I. Abstract framework, Computing, 82 (2008), pp. 103-119.

[10] R. Beck, R. Hiptmair, R. H. Hoppe, and B. Wohlmuth, Residual based a posteriori error estimators for eddy current computation, M2AN Math. Model. Numer. Anal., 34 (2000), pp. 159-182.

Copyright $@$ by SIAM. Unauthorized reproduction of this article is prohibited. 
[11] L. Cao, Y. Zhang, W. Allegretto, And Y. Lin, Multiscale asymptotic method for Maxwell's equations in composite materials, SIAM J. Numer. Anal., 47 (2010), pp. 4257-4289.

[12] V. T. Chu And V. H. Hoang, Homogenization Error for Two Scale Maxwell Equations, arXiv:1512.02788, 2015.

[13] P. Ciarlet, Jr. and C. Stohrer, Finite-element heterogeneous multiscale method for the Helmholtz equation, C. R. Math. Acad. Sci. Paris, 352 (2014), pp. 755-760.

[14] P. Ciarlet, S. Fliss, And C. Stohrer, On the Approximation of Electromagnetic Fields by Edge Finite Elements. Part 2: A Heterogeneous Multiscale Method for Maxwell's Equations, preprint, 2016, https://hal.inria.fr/hal-01364782.

[15] P. ClÉment, Approximation by finite element functions using local regularization, Rev. Française Automat. Informat. Recherche Opérationnelle Sér. Rouge Anal. Numér., 9 (1975), pp. $77-84$.

[16] M. Costabel and M. Dauge, Singularities of electromagnetic fields in polyhedral domains, Arch. Ration. Mech. Anal., 151 (2000), pp. 221-276.

[17] H. Duan, S. Li, R. C. E. TAN, And W. Zheng, A delta-regularization finite element method for a double curl problem with divergence-free constraint, SIAM J. Numer. Anal., 50 (2012), pp. 3208-3230.

[18] W. E, P. Ming, And P. Zhang, Analysis of the heterogeneous multiscale method for elliptic homogenization problems, J. Amer. Math. Soc., 18 (2005), pp. 121-156.

[19] A. L. Efros And A. L. Pokrovsky, Dielectric photonic crystal as medium with negative electric permittivity and magnetic permeability, Solid State Communications, 129 (2004), pp. 643-647.

[20] B. Engquist And W. E, The heterogeneous multiscale methods, Commun. Math. Sci., 1 (2003), pp. 87-132.

[21] B. ENGQUist AND W. E, The heterogeneous multi-scale method for homogenization problems, in Multiscale Methods in Science and Engineering, Lect. Notes Comput. Sci. Eng. 44, Springer, Berlin, 2005, pp. 89-110.

[22] B. Engquist, H. Holst, AND O. Runborg, Multi-scale methods for wave propagation in heterogeneous media, Commun. Math. Sci., 9 (2011), pp. 33-56.

[23] B. Engquist, H. Holst, And O. Runborg, Multiscale methods for wave propagation in heterogeneous media over long time, in Numerical Analysis of Multiscale Computations, Lect. Notes Comput. Sci. Eng. 82, Springer, Berlin, 2012, pp. 167-186.

[24] B. Engquist And O. Runborg, Computational high frequency wave propagation, Acta Numer., 12 (2003), pp. 181-266.

[25] P. Fernandes AND M. RAFFetTo, Existence, uniqueness and finite element approximation of the solution of time-harmonic electromagnetic boundary value problems involving metamaterials, COMPEL, 24 (2005), pp. 1450-1469.

[26] A. GLORIA, An analytical framework for the numerical homogenization of monotone elliptic operators and quasiconvex energies, Multiscale Model. Simul., 5 (2006), pp. 996-1043.

[27] P. Henning, Skript zur Vorlesung Homogenisierung und Mehrskalenmodellierung, www.wwu. de/math/num/Vorlesungen/Mehrskalen_SS12/Skript/running_skript.pdf (2012).

[28] P. Henning and M. Ohlberger, The heterogeneous multiscale finite element method for elliptic homogenization problems in perforated domains, Numer. Math., 113 (2009), pp. 601629.

[29] P. Henning And M. OhlBerger, The heterogeneous multiscale finite element method for advection-diffusion problems with rapidly oscillating coefficients and large expected drift, Netw. Heterog. Media, 5 (2010), pp. 711-744.

[30] P. Henning, M. Ohlberger, and B. Verfürth, Analysis of multiscale methods for timeharmonic Maxwell's equations, Proc. Appl. Math. Mech., 16 (2016), pp 559-560.

[31] L. Jiang And Y. EFEndiev, A priori estimates for two multiscale finite element methods using multiple global fields to wave equations, Numer. Methods Partial Differential Equations, 28 (2012), pp. 1869-1892.

[32] L. Jiang, Y. Efendiev, And V. Ginting, Analysis of global multiscale finite element methods for wave equations with continuum spatial scales, Appl. Numer. Math., 60 (2010), pp. 862876.

[33] J. D. Joannapolous, S. G. Johnson, J. N. Winn, and R. D. Meade, Photonic Crystals: Molding the Flow of Light, 2nd ed., Princeton University Press, Princeton, NJ, 2008; also available online at http://ab-initio.mit.edu/book/photonic-crystals-book.pdf.

[34] D. Lukkassen, G. Nguetseng, and P. Wall, Two-scale convergence, Int. J. Pure Appl. Math., 2 (2002), pp. 35-86.

[35] C. Luo, S. G. Johnson, J. D. Joannopoulos, And J. B. Pendry, All-angle negative refraction without negative effective index, Phys. Rev. B, 65 (2002), 201104.

[36] R. Milk And F. Schindler, Dune-GDT 2015, doi:10.5281/zenodo.35389.

Copyright $@$ by SIAM. Unauthorized reproduction of this article is prohibited. 
[37] P. Monk, Finite Element Methods for Maxwell's Equations, Numer. Math. Sci. Comput., Oxford University Press, Oxford, 2003.

[38] M. Ohlberger, A posteriori error estimates for the heterogeneous multiscale finite element method for elliptic homogenization problems, Multiscale Model. Simul., 4 (2005), pp. 88114.

[39] A. Pokrovsky And A. Efros, Diffraction theory and focusing of light by a slab of left-handed material, Phys. B, 338 (2003), pp. 333-337.

[40] J. SchöBerL, A posteriori error estimates for Maxwell equations, Math. Comp., 77 (2008), pp. 633-649.

[41] J. SCHÖBERL, Numerical Methods for Maxwell Equations, 2009, http://www.asc.tuwien.ac.at/ $\sim_{\text {schoeberl/wiki/lva/notes/maxwell.pdf. }}$

[42] D. R. Smith, J. B. Pendry, and M. C. K. Wiltshire, Metamaterials and negative refractive index, Science, 305 (2004), pp. 788-792.

[43] R. Verfürth, A Posteriori Error Estimation Techniques for Finite Element Methods, Numer. Math. Sci. Comput., Oxford University Press, Oxford, 2013.

[44] A. Visintin, Two-scale convergence of first-order operators, Z. Anal. Anwend., 26 (2007), pp. 133-164.

[45] N. Wellander, Homogenization of the Maxwell equations. Case I. Linear theory, Appl. Math., 46 (2001), pp. 29-51.

[46] N. Wellander, The two-scale Fourier transform approach to homogenization; periodic homogenization in Fourier space, Asymptot. Anal., 62 (2009), pp. 1-40.

[47] N. Wellander and G. Kristensson, Homogenization of the Maxwell equations at fixed frequency, SIAM J. Appl. Math., 64 (2003), pp. 170-195.

[48] S. Zaglmayr, High Order Finite Element Methods for Electromagnetic Field Computation, Ph.D. thesis, Johannes Kepler Universität Linz, 2006.

[49] Y. Zhang, L.-Q. CAO, AND Y.-S. Wong, Multiscale computations for $3 D$ time-dependent Maxwell's equations in composite materials, SIAM J. Sci. Comput., 32 (2010), pp. 25602583.

Copyright (c) by SIAM. Unauthorized reproduction of this article is prohibited. 